\title{
TAIL BEHAVIOR OF MULTIVARIATE LÉVY-DRIVEN MIXED MOVING AVERAGE PROCESSES AND SUPOU STOCHASTIC VOLATILITY MODELS
}

\author{
MARTIN MOSER, ${ }^{*}$ Technische Universität München \\ ROBERT STELZER, ${ }^{* *}$ Ulm University
}

\begin{abstract}
Multivariate Lévy-driven mixed moving average (MMA) processes of the type $X_{t}=$ $\iint f(A, t-s) \Lambda(\mathrm{d} A, \mathrm{~d} s)$ cover a wide range of well known and extensively used processes such as Ornstein-Uhlenbeck processes, superpositions of Ornstein-Uhlenbeck (supOU) processes, (fractionally integrated) continuous-time autoregressive moving average processes, and increments of fractional Lévy processes. In this paper we introduce multivariate MMA processes and give conditions for their existence and regular variation of the stationary distributions. Furthermore, we study the tail behavior of multivariate supOU processes and of a stochastic volatility model, where a positive semidefinite supOU process models the stochastic volatility.
\end{abstract}

Keywords: Lévy basis; mixed moving average process; multivariate regular variation; supOU process; stochastic volatility model; tail behavior

2010 Mathematics Subject Classification: Primary 60G51; 60G70

Secondary $60 \mathrm{H} 07 ; 60 \mathrm{G} 10$

\section{Introduction}

In many areas of application Lévy-driven processes are used for modeling time series. One elementary example of the processes used is the Lévy-driven Ornstein-Uhlenbeck-type (OUtype) process

$$
X_{t}=\int_{-\infty}^{t} \mathrm{e}^{-a(t-s)} \mathrm{d} L_{s},
$$

where $L$ is a Lévy process (see [29] for a detailed introduction). These processes are used, for instance, to model the variance (i.e. the volatility in the terminology of mathematical finance) in the OU-type stochastic volatility model of [2], which has been extended to the multivariate setting in [23]. Even though this model has many nice properties (e.g. stochastic volatility with jumps and clustering, heavy tails, etc.), it does not account for the long-memory effects that can often be found in real data. This problem can be bypassed by the superposition of OU-type processes which leads to supOU processes of the type

$$
X_{t}=\int_{\mathbb{R}} \int_{-\infty}^{t} \mathrm{e}^{a(t-s)} \Lambda(\mathrm{d} a, \mathrm{~d} s),
$$

Received 12 August 2010; revision received 18 July 2011.

* Postal address: TUM Institute for Advanced Study \& Zentrum Mathematik, Technische Universität München, Boltzmannstraße 3, 85748 Garching bei München, Germany. Email address: moser@ma.tum.de

** Postal address: Institute of Mathematical Finance, Ulm University, Helmholtzstraße 18, 89081 Ulm, Germany. Email address: robert.stelzer@uni-ulm.de 
where $\Lambda$ is a so-called Lévy basis. These processes have been introduced in [1], extended to a multivariate setting in [3], and used in the multivariate supOU-type stochastic volatility model of [4]. Bayesian estimation of univariate supOU stochastic volatility models is, e.g. carried out in [13].

The aim of this paper is to analyze the tail behavior of the multivariate mixed moving average (MMA) processes

$$
X_{t}=\int_{M_{d}^{-}} \int_{\mathbb{R}} f(A, t-s) \Lambda(\mathrm{d} A, \mathrm{~d} s)
$$

that allow for a general kernel function $f: M_{d}^{-} \times \mathbb{R} \mapsto M_{n, d}$ ( $\Lambda$ is an $\mathbb{R}^{d}$-valued Lévy basis in this setting). They reach back to [30] and they cover both OU and supOU processes as well as continuous-time autoregressive moving average (CARMA) processes, fractionally integrated CARMA processes (cf. [8] and [20]), and increments of fractional Lévy processes (cf. [6], [19], and the references therein). The tail behavior of univariate MMA processes has already been studied in [10] and [17], and we extend the results to a multivariate setting and also analyze the special case of supOU processes and the related stochastic volatility model given by

$$
\mathrm{d} X_{t}=a_{t} \mathrm{~d} t+\Sigma_{t}^{1 / 2} \mathrm{~d} W_{t}+\Psi\left(\mathrm{d} L_{t}\right), \quad X_{0}=0,
$$

where $a$ is an $\mathbb{R}^{d}$-valued predictable process, $W$ is the standard $d$-dimensional Brownian motion, $L$ is the Lévy process associated with $\Lambda, \Psi: \mathbb{S}_{d} \mapsto \mathbb{R}^{d}$ is a linear operator, and the stochastic volatility process $\left(\Sigma_{t}\right)_{t \in \mathbb{R}}$ is a matrix-valued positive semidefinite supOU process. The multivariate extension is nontrivial, since the definition of regular variation is considerably more involved in the multivariate setting and we have to take the peculiarities created by the use of matrices into account.

In the field of finance the tail behavior is of great importance for risk assessment and risk management. Moreover, our results allow one to understand how to model the so-called 'correlation breakdown' effect (viz. in times of extreme crisis basically all correlations get close to 1 ), which is regarded by econometrics to be typically present in observed financial data.

The paper is structured as follows. We start by giving some general notation in Section 2.1. In Section 2.2 we will give a short excursion to multivariate regular variation that we need when we analyze the tail behavior of the processes given. An introduction to Lévy bases and conditions for the existence of integrals with respect to Lévy bases will be given in Section 2.3. Based on these preliminaries, we then define and analyze multivariate MMA processes in Section 3 . We give sufficient conditions for the MMA processes to be regularly varying given that the driving Lévy basis is regularly varying. Furthermore, we examine the restrictiveness of the conditions by establishing closely related necessary conditions. In Section 4 we apply these results to multivariate supOU processes and give some more accessible conditions for this special case. Finally, we consider a stochastic volatility model that is based on positive semidefinite supOU processes and analyze its tail behavior in Section 5, which is very important for risk assessment.

\section{Preliminaries}

\subsection{Notation}

Given the real numbers $\mathbb{R}$, we use the notation $\mathbb{R}^{+}$for the positive real numbers and $\mathbb{R}^{-}$for the negative real numbers, both without 0 . The Borel sets are denoted by $\mathcal{B}$, where $\mathcal{B}_{\mathrm{b}}$ are the bounded Borel sets and $\mathscr{B}_{\mu}:=\{B \in \mathscr{B}: \mu(\partial B)=0\}$ describes all Borel sets with no $\mu$-mass at the boundary $\partial B$. The closure of a set $B$ is given by $\bar{B}$. We denote by $S$ the unit sphere, by $\lambda$ the Lebesgue measure on $\mathbb{R}$, and by $N\left(0, I_{d}\right)$ the standard normal distribution in $\mathbb{R}^{d}$. 
For matrices, $M_{n, d}$ denotes the set of all $n \times d$ matrices and $M_{d}$ denotes the set of all $d \times d$ matrices; $M_{d}^{-}$is the set of all $d \times d$ matrices with eigenvalues having strictly negative real part. We denote by $I_{d}$ the $d \times d$ identity matrix, by $\mathbb{S}_{d}$ the symmetric $d \times d$ matrices, and by $\mathbb{S}_{d}^{+}$the positive semidefinite $d \times d$ matrices. We write $A^{\top}$ for the transpose of a matrix $A$ and $\|A\|$ for its matrix norm. Since all norms are equivalent, the type of norm is not important for our results, but if we make no further specifications, we use the operator norm induced by the Euclidean norm. Define $j(A):=\min _{\|x\|=1}\|A x\|$, the modulus of injectivity of $A$. The well-known operation vec(A) creates a vector by stacking the columns of an $n \times n$ matrix $A$ below each other to obtain an $\mathbb{R}^{n^{2}}$-valued vector and ' $\otimes$ ' is the tensor product of two matrices. Vague convergence is denoted by ' $\rightarrow$ $\rightarrow$ '. It is defined on the one-point uncompactification $\overline{\mathbb{R}}^{d} \backslash\{0\}$, which ensures that the sets $B \subseteq V_{r}:=\{x:\|x\|>r\}, r>0$, that are bounded away from the origin can be referred to as the relatively compact sets in the vague topology. In this topology, the compact sets will be denoted by $\mathcal{K}$ and the open sets by $q$.

\subsection{Multivariate regular variation}

For the analysis of the tail behavior of multivariate stochastic processes, we use the wellestablished concept of regular variation. However, there is not only one single definition of multivariate regular variation, but many different equivalent definitions. For detailed and very good introductions to the different approaches to multivariate regular variation, we refer the reader to [18] and [27, Section 6]. We start with a well-known definition of multivariate regular variation (cf. [16] and [25]).

Definition 2.1. (Multivariate regular variation.) A random vector $X \in \mathbb{R}^{d}$ is called regularly varying with index $\alpha>0$ if there exist a slowly varying function $l: \mathbb{R} \mapsto \mathbb{R}$ and a nonzero Radon measure $\mu$ defined on $\mathcal{B}\left(\overline{\mathbb{R}}^{d} \backslash\{0\}\right)$ with $\mu\left(\overline{\mathbb{R}}^{d} \backslash \mathbb{R}^{d}\right)=0$ such that, as $u \rightarrow \infty$,

$$
u^{\alpha} l(u) \mathrm{P}\left(u^{-1} X \in \cdot\right) \stackrel{\mathrm{v}}{\rightarrow} \mu(\cdot)
$$

on $\mathcal{B}\left(\overline{\mathbb{R}}^{d} \backslash\{0\}\right)$. We write $X \in \mathrm{RV}(\alpha, l, \mu)$.

Similarly, we call a Radon measure $v$ regularly varying if $\alpha, l$, and $\mu$ exist as above with

$$
u^{\alpha} l(u) v(u \cdot) \stackrel{\mathrm{v}}{\rightarrow} \mu(\cdot)
$$

for $u \rightarrow \infty$, and we write $v \in \mathrm{RV}(\alpha, l, \mu)$.

A stochastic process $\left(X_{t}\right)_{t \in \mathbb{R}} \in \mathbb{R}^{d}$ is called regularly varying with index $\alpha$ if all its finitedimensional distributions are regularly varying with index $\alpha$.

The measure $\mu$ is homogeneous, i.e. it necessarily satisfies the condition

$$
\mu(t B)=t^{-\alpha} \mu(B)
$$

for all $B \in \mathcal{B}\left(\overline{\mathbb{R}}^{d} \backslash\{0\}\right)$ and $t>0$. We make use of this property throughout the paper.

In this paper we will deal with infinitely divisible random variables and processes. For those, the following very useful connection between regular variation of the random variable and its Lévy measure exists.

Theorem 2.1. ([16, Proposition 3.1].) Let $X \in \mathbb{R}^{d}$ be an infinitely divisible random vector with Lévy measure $\nu$. Then $X \in \operatorname{RV}(\alpha, l, \mu)$ if and only if $v \in \operatorname{RV}(\alpha, l, \mu)$.

Furthermore, we will also need regular variation of matrix-valued random variables and processes. If we take into account the well-known vec operation that creates a vector by stacking the columns of a matrix below each other, we can simply apply the above definition. This allows us to use all known results for the $\mathbb{R}^{d}$-valued case also in the matrix-valued case. 


\subsection{Lévy bases and integration}

In this section we recall $\mathbb{R}^{d}$-valued Lévy bases, which are generalizations of Lévy processes, and the related integration theory. For a general introduction to Lévy processes and infinitely divisible distributions, see [29]. Lévy bases are also called infinitely divisible independently scattered random measures in the literature. For more details on Lévy bases, see [22] and [24].

Definition 2.2. (Lévy basis.) An $\mathbb{R}^{d}$-valued random measure $\Lambda=(\Lambda(B))$ with $B \in \mathscr{B}_{\mathrm{b}}\left(M_{d}^{-} \times\right.$ $\mathbb{R})$ is called a Lévy basis if

- the distribution of $\Lambda(B)$ is infinitely divisible for all $B \in \mathcal{B}_{\mathrm{b}}\left(M_{d}^{-} \times \mathbb{R}\right)$,

- for any $n$, the random variables $\Lambda\left(B_{1}\right), \ldots, \Lambda\left(B_{n}\right)$ are independent for pairwise disjoint sets $B_{1}, \ldots, B_{n} \in \mathcal{B}_{\mathrm{b}}\left(M_{d}^{-} \times \mathbb{R}\right)$,

- for any pairwise disjoint sets $\left(B_{i}\right)_{i \in \mathbb{N}} \in \mathcal{B}_{\mathrm{b}}\left(M_{d}^{-} \times \mathbb{R}\right)$ with $\bigcup_{n \in \mathbb{N}} B_{n} \in \mathcal{B}_{\mathrm{b}}\left(M_{d}^{-} \times \mathbb{R}\right)$, we have $\Lambda\left(\bigcup_{n \in \mathbb{N}} B_{n}\right)=\sum_{n \in \mathbb{N}} \Lambda\left(B_{n}\right)$ almost surely.

In this paper we restrict ourselves to time-homogeneous and factorizable Lévy bases, i.e. Lévy bases with characteristic function

$$
\mathrm{E}\left(\mathrm{e}^{\mathrm{i} u^{\top} \Lambda(B)}\right)=\mathrm{e}^{\varphi(u) \Pi(B)}
$$

for all $u \in \mathbb{R}^{d}$ and $B \in \mathcal{B}_{\mathrm{b}}\left(M_{d}^{-} \times \mathbb{R}\right)$, where $\Pi=\lambda \times \pi$ is the product of a probability measure $\pi$ on $M_{d}^{-}(\mathbb{R})$ and the Lebesgue measure $\lambda$ on $\mathbb{R}$ and

$$
\varphi(u)=\mathrm{i} u^{\top} \gamma-\frac{1}{2} u^{\top} \Sigma u+\int_{\mathbb{R}^{d}}\left(\mathrm{e}^{\mathrm{i} u^{\top} x}-1-\mathrm{i} u^{\top} x \mathbf{1}_{[-1,1]}(\|x\|)\right) v(\mathrm{~d} x)
$$

is the cumulant transform of an infinitely divisible distribution with characteristic triplet $(\gamma$, $\Sigma, v)$. By $L$ we denote the underlying Lévy process associated with $(\gamma, \Sigma, \nu)$ and given by $L_{t}=\Lambda\left(M_{d}^{-} \times(0, t]\right)$ and $L_{-t}=\Lambda\left(M_{d}^{-} \times[-t, 0)\right)$ for $t \in \mathbb{R}^{+}$. The quadruple $(\gamma, \Sigma, \nu, \pi)$ determines the distribution of the Lévy basis completely and, therefore, it is called the generating quadruple. A definition of $\mathbb{S}_{d}$-valued Lévy bases follows along the same lines.

Remark 2.1. Considering only time homogeneous and factorizable Lévy bases is motivated by possible applications, where models with too many parameters are of no real help, and the so far developed theory of special cases, particularly the supOU process, where this assumption is also made. However, it should be noted that this assumption is not overly restrictive, because stationarity of a Lévy-driven MMA obviously requires in general a time-homogeneous Lévy basis, i.e. the Lebesgue measure has to be used on the time axis. In our work it appears very natural to consider only stationary cases. Hence, the only possible generalization would be to allow the infinitely divisible distribution to depend on $A \in M_{d}^{-}$. We could have $\varphi(A, u)$ instead of $\varphi(u)$. Then we would also have a characteristic triplet $(\gamma(A), \Sigma(A), v(A, \mathrm{~d} x))$ with $v$ being a 'Lévy kernel' and all our results would have immediate extensions to this case, noting that as far as regular variation is concerned one would have to demand that $\Lambda(B)$ has to be regularly varying for all sets $B$ with the same index $\alpha$ and slowly varying function $l$ (or 'degenerately $\alpha$-regularly varying', i.e. $\left.u^{\alpha} l(u) \mathrm{P}\left(u^{-1} \Lambda(B \in \cdot)\right) \rightarrow 0\right)$, and the measure of regular variation would have to be given via a nontrivial kernel $\mu_{v}(A, \cdot)$. As in the univariate literature (see [10], [11], and [12]), we refrain from stating our results with this level of generality, since it would not add real insight, but lead to overly technical statements not relevant for applications. 
The main focus of this paper, the MMA processes, are defined by integrating over a function $f$ with respect to a Lévy basis. Regarding the existence of these integrals we recall the following multivariate extension of Theorem 2.7 of [24].

Theorem 2.2. Let $\Lambda$ be an $\mathbb{R}^{d}$-valued Lévy basis with characteristic function of the form (2.1), and let $f: M_{d}^{-} \times \mathbb{R} \mapsto M_{n, d}$ be a measurable function. Then $f$ is $\Lambda$-integrable as a limit in probability in the sense of [24] if and only if

$$
\begin{aligned}
& \int_{M_{d}^{-}} \int_{\mathbb{R}} \| f(A, s) \gamma+\int_{\mathbb{R}^{d}} f(A, s) x\left(\mathbf{1}_{[0,1]}(\|f(A, s) x\|)\right. \\
& \left.-\mathbf{1}_{[0,1]}(\|x\|)\right) \nu(\mathrm{d} x) \| \mathrm{d} s \pi(\mathrm{d} A)<\infty, \\
& \int_{M_{d}^{-}} \int_{\mathbb{R}}\left\|f(A, s) \Sigma f(A, s)^{\top}\right\| \mathrm{d} s \pi(\mathrm{d} A)<\infty,
\end{aligned}
$$

and

$$
\int_{M_{d}^{-}} \int_{\mathbb{R}} \int_{\mathbb{R}^{d}}\left(1 \wedge\|f(A, s) x\|^{2}\right) v(\mathrm{~d} x) \mathrm{d} s \pi(\mathrm{d} A)<\infty .
$$

If $f$ is $\Lambda$-integrable, the distribution of $X_{0}=\int_{M_{d}^{-}} \int_{\mathbb{R}^{+}} f(A, s) \Lambda(\mathrm{d} A, \mathrm{~d} s)$ is infinitely divisible with characteristic triplet $\left(\gamma_{\mathrm{int}}, \Sigma_{\mathrm{int}}, \nu_{\mathrm{int}}\right)$ given by

$$
\begin{aligned}
\gamma_{\text {int }}=\int_{M_{d}^{-}} \int_{\mathbb{R}}\left(f(A, s) \gamma+\int_{\mathbb{R}^{d}} f(A, s) x\left(\mathbf{1}_{[0,1]}(\|f(A, s) x\|)\right.\right. \\
\left.\left.-\mathbf{1}_{[0,1]}(\|x\|)\right) v(\mathrm{~d} x)\right) \mathrm{d} s \pi(\mathrm{d} A), \\
\Sigma_{\text {int }}=\int_{M_{d}^{-}} \int_{\mathbb{R}} f(A, s) \Sigma f(A, s)^{\top} \mathrm{d} s \pi(\mathrm{d} A),
\end{aligned}
$$

and

$$
v_{\text {int }}(B)=\int_{M_{d}^{-}} \int_{\mathbb{R}} \int_{\mathbb{R}^{d}} \mathbf{1}_{B}(f(A, s) x) v(\mathrm{~d} x) \mathrm{d} s \pi(\mathrm{d} A) \text { for all Borel sets } B \subseteq \mathbb{R}^{d} .
$$

We now give some more accessible sufficient conditions for the special case of a regularly varying driving Lévy measure $v$. Therefore, we define the set

$$
\mathbb{L}^{\delta}(\lambda \times \pi):=\left\{f: M_{d}^{-} \times \mathbb{R} \mapsto M_{n, d} \text { measurable, } \int_{M_{d}^{-}} \int_{\mathbb{R}}\|f(A, s)\|^{\delta} \mathrm{d} s \pi(\mathrm{d} A)<\infty\right\} .
$$

The following theorem is a multivariate analogue of Proposition 3.1 of [10], which is nontrivial due to the peculiarities arising from the used matrices.

Theorem 2.3. Let $\Lambda$ be a Lévy basis with values in $\mathbb{R}^{d}$ and characteristic quadruple $(\gamma, \Sigma$, $\nu, \pi)$, let $v$ be regularly varying with index $\alpha$, and let $f: M_{d}^{-} \times \mathbb{R} \mapsto M_{n, d}$. Then $f$ is $\Lambda$-integrable in the sense of [24] and $X_{0}$ is well defined and infinitely divisible with the characteristic triplet given in Theorem 2.2, if one of the following conditions hold.

(i) $L_{1}$ is $\alpha$-stable with $\alpha \in(0,2) \backslash\{1\}$ and $f \in \mathbb{L}^{\alpha} \cap \mathbb{L}^{1}$.

(ii) $f$ is bounded and $f \in \mathbb{L}^{\delta}$ for some $\delta<\alpha, \delta \leq 1$.

(iii) $f$ is bounded, $\mathrm{E} L_{1}=0, \alpha>1$, and $f \in \mathbb{L}^{\delta}$ for some $\delta<\alpha, \delta \leq 2$. 
Proof. We will prove the result by validating conditions (2.2), (2.3), and (2.4) given in Theorem 2.2 in each of the three settings.

Part (i). From Theorem 14.3 of [29] we know that in the $\alpha$-stable case $\Sigma=0$, which makes condition (2.3) trivial. Furthermore, there is a finite measure $\theta$ on the unit sphere $S$ such that

$$
v(B)=\int_{S} \int_{0}^{\infty} \frac{\mathbf{1}_{B}(r \xi)}{r^{1+\alpha}} \mathrm{d} r \theta(\mathrm{d} \xi) \quad \text { for } B \in \mathscr{B}^{d} .
$$

For condition (2.2), this yields

$$
\begin{aligned}
\int_{M_{d}^{-}} & \int_{\mathbb{R}}\left\|f(A, s) \gamma+\int_{\mathbb{R}^{d}} f(A, s) x\left(\mathbf{1}_{[0,1]}(\|f(A, s) x\|)-\mathbf{1}_{[0,1]}(\|x\|)\right) v(\mathrm{~d} x)\right\| \mathrm{d} s \pi(\mathrm{d} A) \\
& =\int_{M_{d}^{-}} \int_{\mathbb{R}} \| f(A, s) \gamma+\int_{S} \int_{0}^{\infty} f(A, s) \xi\left(\mathbf{1}_{[0,1]}(\|f(A, s) r \xi\|)\right. \\
& \left.-\mathbf{1}_{[0,1]}(\|r \xi\|)\right) \frac{\mathrm{d} r}{r^{\alpha}} \theta(\mathrm{d} \xi) \| \mathrm{d} s \pi(\mathrm{d} A) \\
& =\int_{M_{d}^{-}} \int_{\mathbb{R}}\left\|f(A, s) \gamma+f(A, s) \int_{S} \xi \int_{1}^{\|f(A, s) \xi\|^{-1}} r^{-\alpha} \mathrm{d} r \theta(\mathrm{d} \xi)\right\| \mathrm{d} s \pi(\mathrm{d} A) \\
& =\int_{M_{d}^{-}} \int_{\mathbb{R}}\left\|f(A, s) \gamma+f(A, s) \int_{S} \xi \frac{1}{1-\alpha}\left(\|f(A, s) \xi\|^{\alpha-1}-1\right) \theta(\mathrm{d} \xi)\right\| \mathrm{d} s \pi(\mathrm{d} A) \\
& \leq \int_{M_{d}^{-}} \int_{\mathbb{R}}\left(\|f(A, s)\| \gamma+\frac{\|f(A, s)\|^{\alpha}}{1-\alpha} \theta(S)+\frac{\|f(A, s)\|}{1-\alpha} \theta(S)\right) \mathrm{d} s \pi(\mathrm{d} A) \\
& <\infty,
\end{aligned}
$$

where we have used $f \in \mathbb{L}^{\alpha} \cap \mathbb{L}^{1}$. For condition (2.4) we obtain

$$
\begin{aligned}
\int_{M_{d}^{-}} & \int_{\mathbb{R}} \int_{\mathbb{R}^{d}}\left(1 \wedge\|f(A, s) x\|^{2}\right) v(\mathrm{~d} x) \mathrm{d} s \pi(\mathrm{d} A) \\
= & \int_{M_{d}^{-}} \int_{\mathbb{R}} \int_{\mathbb{R}^{d}} \mathbf{1}_{\{\|f(A, s) x\| \geq 1\}} v(\mathrm{~d} x) \mathrm{d} s \pi(\mathrm{d} A) \\
& +\int_{M_{d}^{-}} \int_{\mathbb{R}} \int_{\mathbb{R}^{d}}\|f(A, s) x\|^{2} \mathbf{1}_{\{\|f(A, s) x\| \leq 1\}} v(\mathrm{~d} x) \mathrm{d} s \pi(\mathrm{d} A) .
\end{aligned}
$$

The first term on the right-hand side can be bounded by

$$
\begin{aligned}
\int_{M_{d}^{-}} \int_{\mathbb{R}} \int_{\mathbb{R}^{d}} \mathbf{1}_{\{\|f(A, s) x\| \geq 1\}} \nu(\mathrm{d} x) \mathrm{d} s \pi(\mathrm{d} A) & =\frac{1}{\alpha} \int_{M_{d}^{-}} \int_{\mathbb{R}} \int_{S}\|f(A, s) \xi\|^{\alpha} \theta(\mathrm{d} \xi) \mathrm{d} s \pi(\mathrm{d} A) \\
& \leq \frac{\theta(S)}{\alpha} \int_{M_{d}^{-}} \int_{\mathbb{R}}\|f(A, s)\|^{\alpha} \mathrm{d} s \pi(\mathrm{d} A) \\
& <\infty
\end{aligned}
$$


and, for the second term on the right-hand side, we obtain

$$
\begin{aligned}
\int_{M_{d}^{-}} & \int_{\mathbb{R}} \int_{\mathbb{R}^{d}}\|f(A, s) x\|^{2} \mathbf{1}_{\{\|f(A, s) x\| \leq 1\}} \nu(\mathrm{d} x) \mathrm{d} s \pi(\mathrm{d} A) \\
& =\frac{1}{2-\alpha} \int_{M_{d}^{-}} \int_{\mathbb{R}} \int_{S}\|f(A, s) \xi\|^{\alpha} \theta(\mathrm{d} \xi) \mathrm{d} s \pi(\mathrm{d} A) \\
& \leq \frac{\theta(S)}{2-\alpha} \int_{M_{d}^{-}} \int_{\mathbb{R}}\|f(A, s)\|^{\alpha} \mathrm{d} s \pi(\mathrm{d} A) \\
& <\infty
\end{aligned}
$$

Parts (ii) and (iii). Condition (2.3) can be bounded by

$$
\int_{M_{d}^{-}} \int_{\mathbb{R}}\left\|f(A, s) \Sigma f(A, s)^{\top}\right\| \mathrm{d} s \pi(\mathrm{d} A) \leq\|\Sigma\| \int_{M_{d}^{-}} \int_{\mathbb{R}}\|f(A, s)\|^{2} \mathrm{~d} s \pi(\mathrm{d} A)<\infty,
$$

which follows from the boundedness of $f$ together with $f \in \mathbb{L}^{\delta}$ for some $\delta \leq 2$. For condition (2.4), we use (2.5) again. For the first term on the right-hand side of (2.5), we use the inequality

$$
\|f(A, s)\|\|x\| \geq\|f(A, s) x\| \geq 1,
$$

which implies that

This yields

$$
\|x\| \geq \frac{1}{\|f(A, s)\|}
$$

$$
\int_{M_{d}^{-}} \int_{\mathbb{R}} \int_{\mathbb{R}^{d}} \mathbf{1}_{\{\|f(A, s) x\| \geq 1\}} v(\mathrm{~d} x) \mathrm{d} s \pi(\mathrm{d} A) \leq \int_{M_{d}^{-}} \int_{\mathbb{R}} v\left(\left\{\|x\| \geq \frac{1}{\|f(A, s)\|}\right\}\right) \mathrm{d} s \pi(\mathrm{d} A) .
$$

Now we can apply the Potter bounds (see [27, Proposition 2.6(ii)]), giving the existence of some $t_{0}$ such that, for all $t \geq t_{0}$, a regularly varying function (in our case $v$ ) can be bounded. Therefore, we distinguish the cases $1 /\|f(A, s)\|>t_{0}$ and $1 /\|f(A, s)\|<t_{0}$. For the first case, we set $\widetilde{C}:=\sup \left\{\|f(A, s)\|:\|f(A, s)\|<1 / t_{0}\right\} \leq 1 / t_{0}$. Then we can apply the Potter bounds for $t=1 / \widetilde{C} \geq t_{0}$ to obtain

$$
\begin{aligned}
& \int_{M_{d}^{-}} \int_{\mathbb{R}} \mathbf{1}_{\left\{1 /\|f(A, s)\|>t_{0}\right\}} v\left(\left\{\|x\| \geq \frac{1}{\|f(A, s)\|}\right\}\right) \mathrm{d} s \pi(\mathrm{d} A) \\
& \quad \leq(1+\alpha-\delta) \int_{M_{d}^{-}} \int_{\mathbb{R}} \mathbf{1}_{\left\{1 /\|f(A, s)\|>t_{0}\right\}} v\left(\left\{\|x\| \geq \frac{1}{\widetilde{C}}\right\}\right)\left(\frac{\|f(A, s)\|}{\widetilde{C}}\right)^{\delta} \mathrm{d} s \pi(\mathrm{d} A) \\
& \quad<\infty .
\end{aligned}
$$

In the other case we set $C:=\sup \|f(A, s)\|<\infty$ and obtain

$$
\begin{aligned}
\int_{M_{d}^{-}} & \int_{\mathbb{R}} \mathbf{1}_{\left\{1 /\|f(A, s)\| \leq t_{0}\right\}} v\left(\left\{\|x\| \geq \frac{1}{\|f(A, s)\|}\right\}\right) \mathrm{d} s \pi(\mathrm{d} A) \\
& \leq \int_{M_{d}^{-}} \int_{\mathbb{R}} \mathbf{1}_{\left\{1 /\|f(A, s)\| \leq t_{0}\right\}} v\left(\left\{\|x\| \geq \frac{1}{C}\right\}\right) \mathrm{d} s \pi(\mathrm{d} A) \\
& =v\left(\left\{\|x\| \geq \frac{1}{C}\right\}\right) \pi \times \lambda\left(\left\{(A, s):\|f(A, s)\| \geq \frac{1}{t_{0}}\right\}\right) \\
& <\infty
\end{aligned}
$$


since $f \in \mathbb{L}^{\delta}$. The second term on the right-hand side of (2.5) can be bounded by

$$
\begin{aligned}
\int_{M_{d}^{-}} & \int_{\mathbb{R}} \int_{\mathbb{R}^{d}}\|f(A, s) x\|^{2} \mathbf{1}_{\{\|f(A, s) x\| \leq 1\}} v(\mathrm{~d} x) \mathrm{d} s \pi(\mathrm{d} A) \\
= & \int_{M_{d}^{-}} \int_{\mathbb{R}}\left(\int_{\|x\|<1}\|f(A, s) x\|^{2} \mathbf{1}_{\{\|f(A, s) x\| \leq 1\}} v(\mathrm{~d} x)\right. \\
& \left.\quad+\int_{\|x\| \geq 1}\|f(A, s) x\|^{2} \mathbf{1}_{\{\|f(A, s) x\| \leq 1\}} v(\mathrm{~d} x)\right) \mathrm{d} s \pi(\mathrm{d} A) \\
\leq & \int_{M_{d}^{-}} \int_{\mathbb{R}}\|f(A, s)\|^{2} \mathrm{~d} s \pi(\mathrm{d} A) \int_{\|x\|<1}\|x\|^{2} v(\mathrm{~d} x) \\
& +\int_{M_{d}^{-}} \int_{\mathbb{R}}\|f(A, s)\|^{\delta} \mathrm{d} s \pi(\mathrm{d} A) \int_{\|x\| \geq 1}\|x\|^{\delta} v(\mathrm{~d} x) \\
<\infty &
\end{aligned}
$$

where we have used the fact that, for bounded functions $f$, the assumption $f \in \mathbb{L}^{\delta}, \delta<2$, implies that $f \in \mathbb{L}^{2}$. Moreover, note that $\int_{\|x\| \geq 1}\|x\|^{\delta} v(\mathrm{~d} x)<\infty$ by [29, Corollary 25.8], since $0<\delta<\alpha$ and, hence, the underlying Lévy process has a finite $\delta$ th moment. Condition (2.2) in Theorem 2.2 can be reformulated as

$$
\begin{gathered}
\int_{M_{d}^{-}} \int_{\mathbb{R}}\left\|f(A, s) \gamma+\int_{\mathbb{R}^{d}} f(A, s) x\left(\mathbf{1}_{[0,1]}(\|f(A, s) x\|)-\mathbf{1}_{[0,1]}(\|x\|)\right) v(\mathrm{~d} x)\right\| \mathrm{d} s \pi(\mathrm{d} A) \\
=\int_{M_{d}^{-}} \int_{\mathbb{R}} \| f(A, s) \gamma+\int_{\|x\|>1} f(A, s) x \mathbf{1}_{\{\|f(A, s) x\| \leq 1\}} v(\mathrm{~d} x) \\
\quad-\int_{\|x\| \leq 1} f(A, s) x \mathbf{1}_{\{\|f(A, s) x\|>1\}} v(\mathrm{~d} x) \| \mathrm{d} s \pi(\mathrm{d} A) \\
=: T .
\end{gathered}
$$

In part (ii) we use $\|f(A, s)\| \leq C$ and, thus, $T$ can be bounded by

$$
\begin{aligned}
T \leq \int_{M_{d}^{-}} \int_{\mathbb{R}}\|f(A, s)\|^{\delta} & \left(C^{1-\delta}|\gamma|+\int_{\|x\|>1}\|x\|^{\delta} v(\mathrm{~d} x)\right. \\
& \left.+C^{1-\delta} \int_{\|x\| \in(1 / C, 1]}\|x\|^{\delta} v(\mathrm{~d} x)\right) \mathrm{d} s \pi(\mathrm{d} A) .
\end{aligned}
$$

In part (iii), we know that $\gamma=-\int_{\|x\|>1} x v(\mathrm{~d} x)$. Since $\alpha>1$ and $\delta<\alpha$, we can arbitrarily choose a $\xi \in(\delta, \alpha)$ with $\xi>1$. This yields

$$
\begin{aligned}
T & \int_{M_{d}^{-}} \int_{\mathbb{R}} \|-\int_{\|x\|>1} f(A, s) x v(\mathrm{~d} x)+\int_{\|x\|>1} f(A, s) x \mathbf{1}_{\{\|f(A, s) x\| \leq 1\}} v(\mathrm{~d} x) \\
& \quad-\int_{\|x\| \leq 1} f(A, s) x \mathbf{1}_{\{\|f(A, s) x\|>1\}} v(\mathrm{~d} x) \| \mathrm{d} s \pi(\mathrm{d} A) \\
& \leq \int_{M_{d}^{-}} \int_{\mathbb{R}} \int_{\mathbb{R}^{d}}\|f(A, s) x\| \mathbf{1}_{\{\|f(A, s) x\|>1\}} v(\mathrm{~d} x) \mathrm{d} s \pi(\mathrm{d} A) \\
\leq & \int_{M_{d}^{-}} \int_{\mathbb{R}} \int_{\mathbb{R}^{d}}\|f(A, s) x\|^{\xi} \mathbf{1}_{\{\|x\|>1 / C\}} v(\mathrm{~d} x) \mathrm{d} s \pi(\mathrm{d} A)
\end{aligned}
$$




$$
\begin{aligned}
& \leq C^{\xi-\delta} \int_{M_{d}^{-}} \int_{\mathbb{R}}\|f(A, s)\|^{\delta} \mathrm{d} s \pi(\mathrm{d} A) \int_{\|x\|>1 / C}\|x\|^{\xi} v(\mathrm{~d} x) \\
& <\infty
\end{aligned}
$$

\section{MMA processes}

MMA processes were first introduced in [30] in the univariate stable case. As already mentioned, they are integrals over a given kernel function with respect to a Lévy basis.

Definition 3.1. (MMA process.) Let $\Lambda$ be an $\mathbb{R}^{d}$-valued Lévy basis on $M_{d}^{-} \times \mathbb{R}$, and let $f: M_{d}^{-} \times \mathbb{R} \mapsto M_{n, d}$ be a measurable function (kernel function). If the process

$$
X_{t}:=\int_{M_{d}^{-}} \int_{\mathbb{R}} f(A, t-s) \Lambda(\mathrm{d} A, \mathrm{~d} s)
$$

exists in the sense of Theorem 2.2 for all $t \in \mathbb{R}$, it is called an $n$-dimensional mixed moving average process (MMA process).

Note that we could also define 'generalized MMA' processes by integrating over a slightly more general function $g: M_{d}^{-} \times \mathbb{R} \times \mathbb{R} \mapsto M_{n, d}$, which gives us

$$
X_{t}=\int_{M_{d}^{-}} \int_{\mathbb{R}} g(A, t, s) \Lambda(\mathrm{d} A, \mathrm{~d} s) .
$$

However, the extension of all upcoming results is trivial, so we stated the results for the notationally easier case of Definition 3.1. Moreover, an MMA process is obviously always stationary and this needs not be true for generalized MMA processes. Note also that $M_{d}^{-}$can obviously be replaced by $M_{d}$ or basically any other Borel set. Again, we state everything for $M_{d}^{-}$, because this eases notation and is the canonical choice in the supOU case.

Existence of the MMA processes follows directly from Theorem 2.2 and Theorem 2.3. Theorem 2.3 turns out to be very useful in this setting, since it is based on similar conditions compared to the key conditions of Theorem 3.1 below: regular variation of the driving Lévy measure $v$ and $f \in \mathbb{L}^{\alpha}(\lambda \times \pi)$.

Theorem 3.1 is the multivariate analog of Equation (3.1) in Proposition 3.2 of [10], where the same conditions, simplified to the univariate setup, are used. A similar result also exists for the special case of a univariate filtered Lévy process, where the kernel function $f$ is continuous and of compact support; see [15, Theorem 22].

Theorem 3.1. Let $\Lambda$ be an $\mathbb{R}^{d}$-valued Lévy basis on $M_{d}^{-} \times \mathbb{R}$ with generating quadruple $(\gamma, \Sigma, v, \pi)$, and let $v \in \mathrm{RV}\left(\alpha, l, \mu_{v}\right)$. If $X_{0}=\int_{M_{d}^{-}} \int_{\mathbb{R}^{+}}^{a} f(A, s) \Lambda(\mathrm{d} A, \mathrm{~d} s)$ exists (in the sense of Theorem 2.2), $f \in \mathbb{L}^{\alpha}(\lambda \times \pi)$, and $\mu_{v}\left(f^{-1}(A, s)\left(\mathbb{R}^{n} \backslash\{0\}\right)\right)=0$ does not hold for $\pi \times \lambda$ almost-every $(A, s)$, then $X_{0} \in \operatorname{RV}\left(\alpha, l, \mu_{X}\right)$ with

$$
\mu_{X}(B):=\int_{M_{d}^{-}} \int_{\mathbb{R}} \int_{\mathbb{R}^{d}} \mathbf{1}_{B}(f(A, s) x) \mu_{\nu}(\mathrm{d} x) \mathrm{d} s \pi(\mathrm{d} A) .
$$

Proof. From Theorem 2.2 we know that the distribution of $X$ is infinitely divisible. Following Theorem 2.1, it is sufficient to prove that its Lévy measure $v_{X}$ is regularly varying. The concrete representation

$$
v_{X}=\int_{M_{d}^{-}} \int_{\mathbb{R}} \int_{\mathbb{R}^{d}} \mathbf{1}_{B}(f(A, s) x) v(\mathrm{~d} x) \mathrm{d} s \pi(\mathrm{d} A)
$$


is also known from Theorem 2.2. Regular variation of $v$ then yields the existence of a constant $\alpha>0$, a slowly varying function $l$, and a Radon measure $\mu_{v}$ on $\mathscr{B}\left(\overline{\mathbb{R}}^{d} \backslash\{0\}\right)$ with $\mu_{v}\left(\overline{\mathbb{R}}^{d} \backslash \mathbb{R}^{d}\right)=0$ such that, as $u \rightarrow \infty$,

$$
u^{\alpha} l(u) v(u \cdot) \stackrel{\mathrm{v}}{\rightarrow} \mu_{v}(\cdot)
$$

Using [27, Theorem 3.2] and Fatou's lemma, we have, for all compact sets $B \in \mathcal{K}$,

$$
\begin{aligned}
& \limsup _{u \rightarrow \infty} u^{\alpha} l(u) \int_{M_{d}^{-}} \int_{\mathbb{R}} \int_{\mathbb{R}^{d}} \mathbf{1}_{u B}(f(A, s) x) v(\mathrm{~d} x) \mathrm{d} s \pi(\mathrm{d} A) \\
& \quad \leq \int_{M_{d}^{-}} \int_{\mathbb{R}} \limsup _{u \rightarrow \infty} u^{\alpha} l(u) \int_{\mathbb{R}^{d}} \mathbf{1}_{u B}(f(A, s) x) v(\mathrm{~d} x) \mathrm{d} s \pi(\mathrm{d} A) \\
& \quad \leq \int_{M_{d}^{-}} \int_{\mathbb{R}} \int_{\mathbb{R}^{d}} \mathbf{1}_{B}(f(A, s) x) \mu_{v}(\mathrm{~d} x) \mathrm{d} s \pi(\mathrm{d} A)
\end{aligned}
$$

and, conversely, for all open sets $B \in \mathcal{G}$ that are relatively compact,

$$
\begin{aligned}
& \liminf _{u \rightarrow \infty} u^{\alpha} l(u) \int_{M_{d}^{-}} \int_{\mathbb{R}} \int_{\mathbb{R}^{d}} \mathbf{1}_{u B}(f(A, s) x) v(\mathrm{~d} x) \mathrm{d} s \pi(\mathrm{d} A) \\
& \geq \int_{M_{d}^{-}} \int_{\mathbb{R}} \liminf _{u \rightarrow \infty} u^{\alpha} l(u) \int_{\mathbb{R}^{d}} \mathbf{1}_{u B}(f(A, s) x) v(\mathrm{~d} x) \mathrm{d} s \pi(\mathrm{d} A) \\
& \geq \int_{M_{d}^{-}} \int_{\mathbb{R}} \int_{\mathbb{R}^{d}} \mathbf{1}_{B}(f(A, s) x) \mu_{v}(\mathrm{~d} x) \mathrm{d} s \pi(\mathrm{d} A) .
\end{aligned}
$$

Note here that, for any set $B \in \mathcal{K}$ (respectively $\mathcal{G}$ ), also the preimage $f(A, s)^{-1}(B) \in \mathcal{K}$ (respectively $g$ ) for all $A, s$, since $f(A, s)$ is, for fixed $A, s$, a linear mapping. This yields the vague convergence

$$
\begin{aligned}
u^{\alpha} l(u) v_{X}(u \cdot) & =u^{\alpha} l(u) \int_{M_{d}^{-}} \int_{\mathbb{R}} \int_{\mathbb{R}^{d}} \mathbf{1}_{u} \cdot(f(A, s) x) v(\mathrm{~d} x) \mathrm{d} s \pi(\mathrm{d} A) \\
& \stackrel{\mathrm{v}}{\rightarrow} \int_{M_{d}^{-}} \int_{\mathbb{R}} \int_{\mathbb{R}^{d}} \mathbf{1}_{\{\cdot\}}(f(A, s) x) \mu_{v}(\mathrm{~d} x) \mathrm{d} s \pi(\mathrm{d} A) \\
& =\mu_{X}(\cdot) .
\end{aligned}
$$

It remains to prove that $\mu_{X}$ is again a Radon measure with $\mu_{X}\left(\overline{\mathbb{R}}^{n} \backslash \mathbb{R}^{n}\right)=0$. The second property follows directly from the observation that

$$
\mathbf{1}_{\left(\overline{\mathbb{R}}^{n} \backslash \mathbb{R}^{n}\right)}(f(A, s) x) \leq \mathbf{1}_{\left(\overline{\mathbb{R}}^{n} \backslash \mathbb{R}^{n}\right)}(x) .
$$

For the local finiteness of $\mu_{X}$, take some compact $B \in \mathcal{B}\left(\overline{\mathbb{R}}^{d} \backslash\{0\}\right)$, i.e. there exists some finite $r>0$ such that $B \subseteq V_{r}:=\{x:\|x\|>r\}$. For all $x$ with $f(A, s) x \in B \subseteq V_{r}$, we have $r<\|f(A, s) x\| \leq\|f(A, s)\|\|x\|$. By using $f \in \mathbb{L}^{\alpha}(\lambda \times \pi)$ and the local finiteness of $\mu_{\nu}$, we 
obtain

$$
\begin{aligned}
\mu_{X}(B) & \leq \int_{M_{d}^{-}} \int_{\mathbb{R}} \int_{\mathbb{R}^{d}} \mathbf{1}_{(r, \infty)}(\|f(A, s)\|\|x\|) \mu_{v}(\mathrm{~d} x) \mathrm{d} s \pi(\mathrm{d} A) \\
& =\int_{M_{d}^{-}} \int_{\mathbb{R}} \mu_{v}\left(\left\{x:\|x\| \geq\|f(A, s)\|^{-1} r\right\}\right) \mathbf{1}_{\mathbb{R} \backslash\{0\}}(\|f(A, s)\|) \mathrm{d} s \pi(\mathrm{d} A) \\
& =\mu_{v}\left(V_{r}\right) \int_{M_{d}^{-}} \int_{\mathbb{R}}\|f(A, s)\|^{\alpha} \mathrm{d} s \pi(\mathrm{d} A) \\
& <\infty .
\end{aligned}
$$

The theorem shows that the tail behavior of the driving Lévy measure determines the tail behavior of the MMA process. Since the Lévy measure is related only to the jumps of the underlying Lévy process, we see that the regular variation of the MMA process is caused by the jumps of the underlying Lévy process. Furthermore, we intuitively know that the extremes of the MMA process are caused by a single extremely big jump in the Lévy basis.

Remark 3.1. Another important consequence of Theorem 3.1 is that we know the concrete measure $\mu_{X}$ of regular variation. This is useful to describe the location or mass of the extremes in $\mathbb{R}^{n}$. It is similar to the spectral measure in an analogue definition of regular variation; see Theorem 1.15(ii) of [18]. See also Example 4.1 below for some calculations of these measures in the OU case.

As mentioned, Theorem 3.1 uses two crucial conditions. The first condition is the regular variation of the driving Lévy measure, meaning that the tail behavior of the input determines the tail behavior of the resulting MMA process. The second condition $f \in \mathbb{L}^{\alpha}(\lambda \times \pi)$ is a restriction on the function $f$. We will now analyze its restrictiveness by looking at necessary conditions. Therefore, we define the set

$$
\mathbb{J}^{\alpha}(\lambda \times \pi):=\left\{f: M_{d}^{-} \times \mathbb{R} \mapsto M_{n, d} \text { measurable, } \int_{M_{d}^{-}} \int_{\mathbb{R}} j(f(A, s))^{\alpha} \mathrm{d} s \pi(\mathrm{d} A)<\infty\right\},
$$

where $j(A)$ is the modulus of injectivity of $A$.

The following theorem extends the univariate work in [10] and [15], where necessary conditions are not considered. Note that our focus is on necessary conditions on $f$, whereas Jacobsen et al. [17] considered whether regular variation of a moving average implies regular variation of the driving Lévy process in the univariate case.

Theorem 3.2. Let $\Lambda$ be an $\mathbb{R}^{d}$-valued Lévy basis on $M_{d}^{-} \times \mathbb{R}$ with generating quadruple $(\gamma, \Sigma, v, \pi)$, and let $v \in \operatorname{RV}\left(\alpha, l, \mu_{\nu}\right)$. If $X_{0}=\int_{M_{d}^{-}} \int_{\mathbb{R}^{+}} f(A, s) \Lambda(\mathrm{d} A, \mathrm{~d} s)$ exists and $\mu_{v}\left(f^{-1}(A, s)\left(\mathbb{R}^{n} \backslash\{0\}\right)\right)=0$ does not hold for $\pi \times \lambda$ almost-every $(A, s)$, then $f \in \mathbb{J}^{\alpha}(\lambda \times \pi)$ is a necessary condition for $X_{0} \in \mathrm{RV}\left(\alpha, l, \mu_{X}\right)$ with

$$
\mu_{X}(B):=\int_{M_{d}^{-}} \int_{\mathbb{R}} \int_{\mathbb{R}^{d}} \mathbf{1}_{B}(f(A, s) x) \mu_{\nu}(\mathrm{d} x) \mathrm{d} s \pi(\mathrm{d} A) .
$$

Proof. We use a simple contradiction. Suppose that $f \notin \mathbb{J}^{\alpha}(\lambda \times \pi)$, i.e.

$$
\int_{M_{d}^{-}} \int_{\mathbb{R}} j(f(A, s))^{\alpha} \mathrm{d} s \pi(\mathrm{d} A)=\infty
$$


Since $\mu_{v}$ is nonzero, there is a positive number $r>0$ such that $\mu_{v}\left(V_{r}\right)>0$. Then we use the relation

$$
j(f(A, s)) \leq \frac{\|f(A, s) x\|}{\|x\|}
$$

for all $x \in \mathbb{R}^{d}$ and obtain

$$
\begin{aligned}
\mu_{X}\left(V_{r}\right) & =\int_{M_{d}^{-}} \int_{\mathbb{R}} \int_{\mathbb{R}^{d}} \mathbf{1}_{V_{r}}(f(A, s) x) \mu_{v}(\mathrm{~d} x) \mathrm{d} s \pi(\mathrm{d} A) \\
& \geq \int_{M_{d}^{-}} \int_{\mathbb{R}} \int_{\mathbb{R}^{d}} \mathbf{1}_{(r, \infty)}(j(f(A, s))\|x\|) \mu_{v}(\mathrm{~d} x) \mathrm{d} s \pi(\mathrm{d} A) \\
& =\mu_{\nu}\left(V_{r}\right) \int_{M_{d}^{-}} \int_{\mathbb{R}} j(f(A, s))^{\alpha} \mathrm{d} s \pi(\mathrm{d} A) \\
& =\infty
\end{aligned}
$$

and this is a contradiction to $\mu_{X}$ being a Radon measure.

Now we have necessary conditions as well as sufficient conditions and both lie close together. Since $j(f(A, s)) \leq\|f(A, s)\|$, we immediately have $\mathbb{L}^{\alpha}(\lambda \times \pi) \subseteq \mathbb{J}^{\alpha}(\lambda \times \pi)$. In the univariate case we even have $\mathbb{L}^{\alpha}(\lambda \times \pi)=\mathbb{J}^{\alpha}(\lambda \times \pi)$ and, thus, we obtain necessary and sufficient conditions.

Having proved the regular variation of the random vector, we can now easily obtain the regular variation of the process $X_{t}$.

Corollary 3.1. Given the conditions of Theorem 3.1, the MMA process $\left(X_{t}\right)_{t \in \mathbb{R}}$ is also regularly varying with index $\alpha$ as a process.

Proof. We have to show that the results also hold for the finite-dimensional distributions of $X_{t}$. For $m \in \mathbb{N}$ and $\boldsymbol{t}=\left(t_{1}, \ldots, t_{m}\right) \in \mathbb{R}^{m}$, we have

$$
\begin{aligned}
\left(\begin{array}{c}
X_{t_{1}} \\
\vdots \\
X_{t_{m}}
\end{array}\right) & =\left(\begin{array}{c}
\int_{M_{d}^{-}} \int_{\mathbb{R}} f\left(A, t_{1}-s\right) \Lambda(\mathrm{d} A, \mathrm{~d} s) \\
\vdots \\
\int_{M_{d}^{-}} \int_{\mathbb{R}} f\left(A, t_{m}-s\right) \Lambda(\mathrm{d} A, \mathrm{~d} s)
\end{array}\right) \\
& =\int_{M_{d}^{-}} \int_{\mathbb{R}}\left(\begin{array}{c}
f\left(A, t_{1}-s\right) \\
\vdots \\
f\left(A, t_{m}-s\right)
\end{array}\right) \Lambda(\mathrm{d} A, \mathrm{~d} s) \\
& =\int_{M_{d}^{-}} \int_{\mathbb{R}} g(A, \boldsymbol{t}, s) \Lambda(\mathrm{d} A, \mathrm{~d} s)
\end{aligned}
$$

with the function $g: M_{d}^{-} \times \mathbb{R}^{m} \times \mathbb{R} \mapsto M_{n m, d}$ defined by

$$
g(A, \boldsymbol{t}, s):=\left(\begin{array}{c}
f\left(A, t_{1}-s\right) \\
\vdots \\
f\left(A, t_{m}-s\right)
\end{array}\right) .
$$

Next we show that $f \in \mathbb{L}^{\beta}(\lambda \times \pi)$ implies that $g \in \mathbb{L}^{\beta}(\lambda \times \pi)$ for all $\beta>0$. Therefore, we choose the matrix norm

$$
\|A\|:=\max _{i, j}\left\{\left|a_{i j}\right|\right\} .
$$


We obtain

$$
\begin{aligned}
\int_{M_{d}^{-}} & \int_{\mathbb{R}}\|g(A, \boldsymbol{t}, s)\|^{\beta} \mathrm{d} s \pi(\mathrm{d} A) \\
& =\int_{M_{d}^{-}} \int_{\mathbb{R}}\left\|\left(\begin{array}{c}
f\left(A, t_{1}-s\right) \\
\vdots \\
f\left(A, t_{m}-s\right)
\end{array}\right)\right\|^{\beta} \mathrm{d} s \pi(\mathrm{d} A) \\
& =\int_{M_{d}^{-}} \int_{\mathbb{R}} \max \left\{\left\|f\left(A, t_{1}-s\right)\right\|, \ldots,\left\|f\left(A, t_{m}-s\right)\right\|\right\}^{\beta} \mathrm{d} s \pi(\mathrm{d} A) \\
& \leq \int_{M_{d}^{-}} \int_{\mathbb{R}}\left\|f\left(A, t_{1}-s\right)\right\|^{\beta}+\cdots+\left\|f\left(A, t_{m}-s\right)\right\|^{\beta} \mathrm{d} s \pi(\mathrm{d} A) \\
& <\infty,
\end{aligned}
$$

since $f \in \mathbb{L}^{\beta}(\lambda \times \pi)$.

If the existence of $X_{t}$ is ensured by Theorem 2.3(ii) or (iii), this implies that, for the existence and regular variation of $\left(X_{t_{1}}^{\top}, \ldots, X_{t_{m}}^{\top}\right)^{\top}$, simple applications of Theorem 3.1 and Theorem 2.3 conclude. However, in general we note that assuming existence of $X_{t}$ in the sense of Theorem 2.2 implies that each of the $m$ individual integrals of $\left(X_{t_{1}}^{\top}, \ldots, X_{t_{m}}^{\top}\right)^{\top}$ exists as a limit of approximating sums in probability. From these individual approximating sums we easily construct a sequence of approximating sums for

$$
\int_{M_{d}^{-}} \int_{\mathbb{R}}\left\|\left(f\left(A, t_{1}-s\right)^{\top}, \ldots, f\left(A, t_{m}-s\right)^{\top}\right)^{\top}\right\|^{\beta} \Lambda(\mathrm{d} A, \mathrm{~d} s)
$$

converging in probability. Hence, the necessary and sufficient existence conditions of Theorem 2.2 are satisfied and Theorem 3.1 shows the regular variation of $\left(X_{t_{1}}^{\top}, \ldots, X_{t_{m}}^{\top}\right)^{\top}$.

A very important class of heavy-tailed distributions are $\alpha$-stable distributions with $\alpha \in(0,2)$. See [28] for a detailed introduction. In Theorem 2.3 we have already given a criterion for the existence of MMA processes with a stable driving Lévy process. Similarly to Theorem 3.1, there is also a well-known link between stability of the driving Lévy measure and stability of the MMA process.

Lemma 3.1. If the driving Lévy process of an MMA process $X_{t}$ is $\alpha$-stable and its Lévy measure is nondegenerate, then $X_{t}$ is also $\alpha$-stable.

Proof. From Theorem 14.3 of [29] we have the result that $\alpha$-stability of an infinitely divisible distribution is equivalent to

$$
\Sigma=0 \quad \text { and } \quad v(\cdot)=b^{-\alpha} v\left(b^{-1} \cdot\right) \quad \text { for all } b>0
$$

Using the assumption together with Theorem 2.2, we immediately have $\Sigma_{X_{t}}=0$ and $v_{X_{t}}(\cdot)=$ $b^{-\alpha} v_{X_{t}}\left(b^{-1} \cdot\right)$.

Now we apply this result to multivariate CARMA (MCARMA) processes. 
Example 3.1. (MCARMA processes.) Univariate Lévy-driven CARMA processes have been introduced in [7], and have been extended to MCARMA processes in [21]. A $d$-dimensional $\operatorname{MCARMA}(p, q)$ process, $p>q$, driven by a two-sided square integrable Lévy process $\left(L_{t}\right)_{t \in \mathbb{R}}$ with $\mathrm{E}\left(L_{1}\right)=0$ and $\mathrm{E}\left(L_{1} L_{1}^{\top}\right)=\Sigma_{L}$ can be formally interpreted as the stationary solution to the $p$ th order $d$-dimensional differential equation

$$
P(D) Y_{t}=Q(D) D L_{t},
$$

where $D$ denotes the differentiation operator with respect to $t$. The autoregressive and moving average polynomials are given by

$$
P(z)=I_{d} z^{p}+A_{1} z^{p-1}+\cdots+A_{p} \quad \text { and } \quad Q(z)=B_{0} z^{q}+B_{1} z^{q-1}+\cdots+B_{q}
$$

with $A_{1}, \ldots, A_{p}, B_{0}, \ldots, B_{q} \in M_{d}$ such that $B_{q} \neq 0$ and $\{z \in \mathbb{C}: \operatorname{det}(P(z))=0\} \subset$ $\mathbb{R} \backslash\{0\}+\mathrm{i} \mathbb{R}$.

The MCARMA process $Y_{t}$ can be represented as a moving average process

$$
Y_{t}=\int_{\mathbb{R}} f(t-s) \mathrm{d} L_{s}
$$

with kernel function $f: \mathbb{R} \mapsto M_{d}$ given by

$$
f(t)=\frac{1}{2 \pi} \int_{\mathbb{R}} \mathrm{e}^{\mathrm{i} u t} P(\mathrm{i} u)^{-1} Q(\mathrm{i} u) \mathrm{d} u .
$$

Obviously, MCARMA processes are MMA processes and, thus, we can apply Lemma 3.1 to obtain an $\alpha$-stable MCARMA process by using an $\alpha$-stable driving Lévy process. Furthermore, by Proposition 3.32 of [21] we know that in the case $p>q+1$ MCARMA processes have continuous sample paths, which are $p-q-1$ times differentiable. This shows that in the case $\alpha \in(0,2)$ and $p>q+1$ we can get heavy-tailed MCARMA processes, where the heavy tails come from the jumps of the underlying Lévy process, but the paths of the observed process are continuous and may even be differentiable.

To illustrate this, we simulated several univariate CARMA $(3,1)$ processes. They are given by the autoregressive and moving average polynomials

$$
p(z)=z^{3}+4.5 z^{2}+6.5 z+3 \text { and } q(z)=z .
$$

The CARMA $(3,1)$ process can then be given in its state space representation (see [21, Theorem 3.12])

$$
G(t)=\int_{-\infty}^{t} \mathrm{e}^{A(t-u)} \beta \mathrm{d} L_{u},
$$

where

$$
A=\left(\begin{array}{ccc}
0 & 1 & 0 \\
0 & 0 & 1 \\
-3 & -6.5 & -4.5
\end{array}\right) \quad \text { and } \beta=\left(\begin{array}{c}
0 \\
1 \\
-4.5
\end{array}\right)
$$

This representation has the advantage that it applies also in the multivariate setting and it directly includes the derivatives of the CARMA process, as long as they exist. In our case, we have $Y_{t}=G_{1}(t)$ and $\mathrm{d} Y_{t} / \mathrm{d} t=G_{2}(t)$. Owing to our foregoing results, $G$ is regularly varying with index $\alpha$ (respectively $\alpha$-stable), if $L$ is. 
For the driving Lévy process $L_{t}$, we used a symmetric $\alpha$-stable Lévy motion without skewness and with $\alpha$-values of 2 (Brownian motion), 1.5, and 1 (both heavy tailed). Furthermore, we plotted the simulated values after a burn-in period of 1000 to ensure stationarity. In all three cases, we can see nicely how the tail behavior of the driving Lévy process determines the tail behavior of the continuous CARMA $(3,1)$ process. In Figure 1 we see the case $\alpha=2$, where the integrator is a light-tailed Brownian motion and the resulting CARMA process is also light tailed. In the cases $\alpha=1.5$ (see Figure 2) and $\alpha=1$ (see Figure 3) the driving process is heavy tailed and, as $\alpha$ is decreasing, the process is more and more determined by only a few very large jumps. The respective CARMA process is also heavy tailed and oscillates around the mean except for some large, but continuous shocks. For these two cases we also plotted the first derivatives of the paths of the CARMA process, which are not continuous anymore, but jointly $\alpha$-stable together with the process itself.
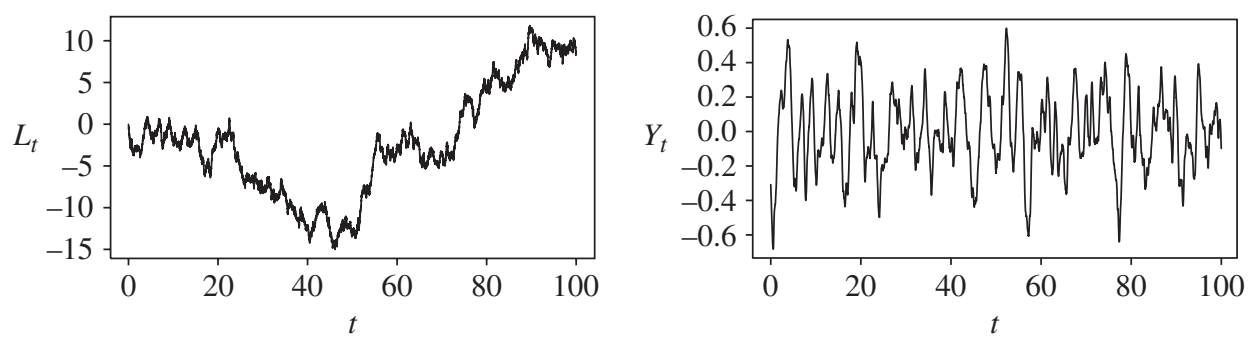

FIGURE 1: Simulations of one path of the driving Lévy process $L_{t}$ and the CARMA $(3,1)$ process $Y_{t}$ in the $\alpha$-stable case with $\alpha=2$.
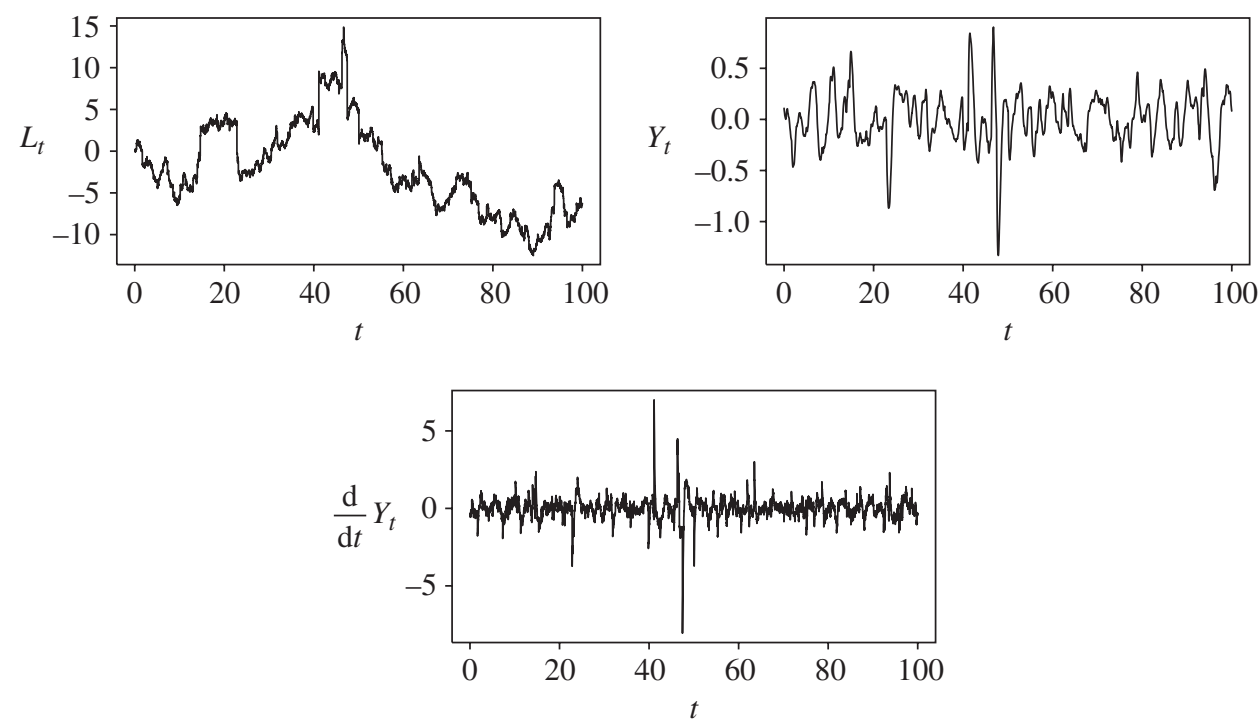

FIGURE 2: Simulations of one path of the driving Lévy process $L_{t}$, the CARMA $(3,1)$ process $Y_{t}$, and its derivative in the $\alpha$-stable case with $\alpha=1.5$. 

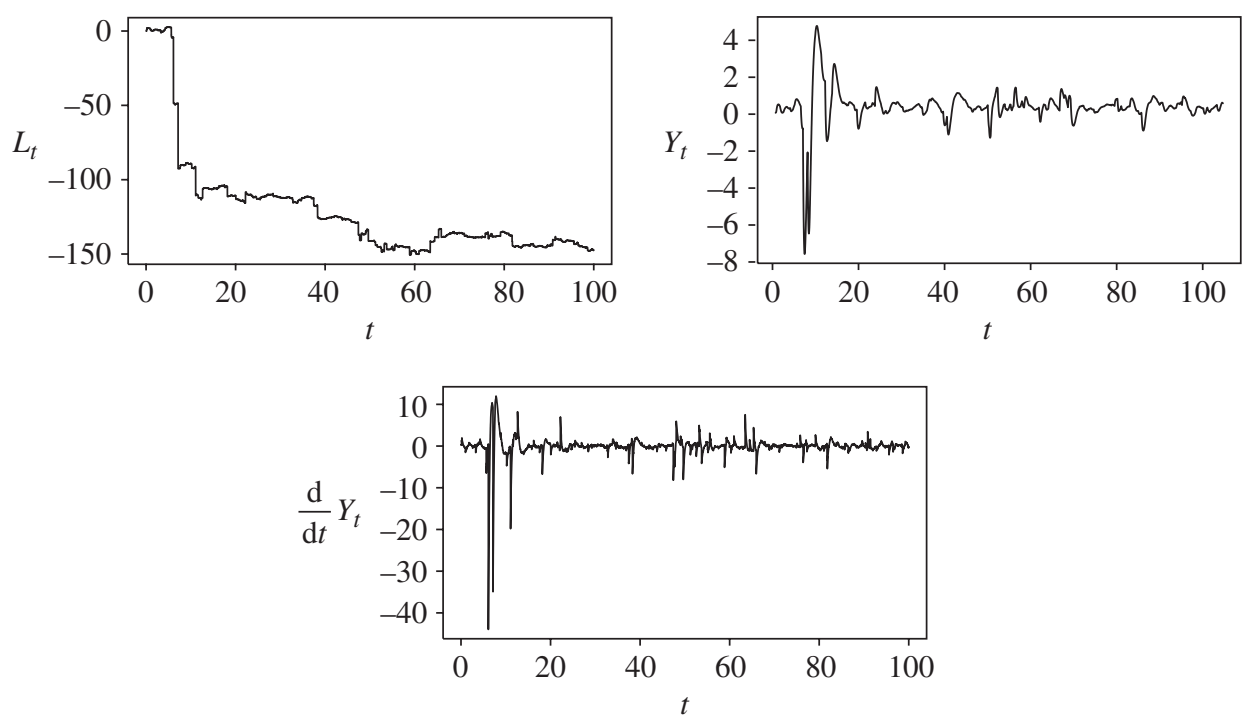

FIGURE 3: Simulations of one path of the driving Lévy process $L_{t}$, the CARMA $(3,1)$ process $Y_{t}$, and its derivative in the $\alpha$-stable case with $\alpha=1$.

\section{Application to supOU processes}

One example of MMA processes are supOU processes. They are especially useful in modeling the stochastic volatility in continuous-time models or long-range dependent time series. For an introduction to univariate supOU processes, see [1], and for the extension to multivariate supOU processes, we refer the reader to [3].

Definition 4.1. ( $\mathbb{R}^{d}$-valued supOU process.) Let $\Lambda$ be an $\mathbb{R}^{d}$-valued Lévy basis on $M_{d}^{-} \times \mathbb{R}$. If the process

$$
X_{t}:=\int_{M_{d}^{-}} \int_{-\infty}^{t} \mathrm{e}^{A(t-s)} \Lambda(\mathrm{d} A, \mathrm{~d} s)
$$

exists for all $t \in \mathbb{R}$, it is called an $\mathbb{R}^{d}$-valued supOU process.

We easily see that supOU processes are MMA processes with special kernel function

$$
f(A, s)=\mathrm{e}^{A s} \mathbf{1}_{[0, \infty)}(s) .
$$

Consequently, the existence of supOU processes is covered by Theorem 2.2. But if we take the special properties of supOU processes into account, some more accessible sufficient conditions for the existence can be given.

Theorem 4.1. ([3, Theorem 3.1].) Let $X_{t}$ be an $\mathbb{R}^{d}$-valued supOU process as defined in Definition 4.1. If

$$
\int_{\|x\|>1} \ln (\|x\|) v(\mathrm{~d} x)<\infty
$$

and there exist measurable functions $\rho: M_{d}^{-} \mapsto \mathbb{R}^{+} \backslash\{0\}$ and $\kappa: M_{d}^{-} \mapsto[1, \infty)$ such that

$$
\left\|\mathrm{e}^{A s}\right\| \leq \kappa(A) \mathrm{e}^{-\rho(A) s} \quad \text { for all } s \in \mathbb{R}^{+}, \pi \text {-almost surely }
$$


and

$$
\int_{M_{d}^{-}} \frac{\kappa(A)^{2}}{\rho(A)} \pi(\mathrm{d} A)<\infty,
$$

then the supOU process $X_{t}=\int_{M_{d}^{-}} \int_{-\infty}^{t} \mathrm{e}^{A(t-s)} \Lambda(\mathrm{d} A, \mathrm{~d} s)$ is well defined for all $t \in \mathbb{R}$ and stationary. Furthermore, the stationary distribution of $X_{t}$ is infinitely divisible with characteristic triplet $\left(\gamma_{X}, \Sigma_{X}, v_{X}\right)$ given by Theorem 2.2.

Now we want to go one step further and analyze the tail behavior, but regular variation of the supOU processes follows directly from Theorem 3.1.

Corollary 4.1. Let $\Lambda \in \mathbb{R}^{d}$ be a Lévy basis on $M_{d}^{-} \times \mathbb{R}$ with generating quadruple $(\gamma, \Sigma, \nu$, $\pi)$, and let $\nu \in \operatorname{RV}\left(\alpha, l, \mu_{\nu}\right)$. If the conditions of Theorem 4.1 hold and, additionally,

$$
\int_{M_{d}^{-}} \frac{\kappa(A)^{\alpha}}{\rho(A)} \pi(\mathrm{d} A)<\infty,
$$

then $X_{0}=\int_{M_{d}^{-}} \int_{\mathbb{R}^{+}} \mathrm{e}^{A s} \Lambda(\mathrm{d} A, \mathrm{~d} s) \in \mathrm{RV}\left(\alpha, l, \mu_{X}\right)$ with Radon measure

$$
\mu_{X}(\cdot):=\int_{M_{d}^{-}} \int_{\mathbb{R}^{+}} \int_{\mathbb{R}^{d}} \mathbf{1}_{\{\cdot\}}\left(\mathrm{e}^{A s} x\right) \mu_{v}(\mathrm{~d} x) \mathrm{d} s \pi(\mathrm{d} A) .
$$

Proof. Using the given conditions, we have

$$
\begin{aligned}
\int_{M_{d}^{-}} \int_{\mathbb{R}^{+}}\left\|\mathrm{e}^{A s}\right\|^{\alpha} \mathrm{d} s \pi(\mathrm{d} A) & \leq \int_{M_{d}^{-}} \int_{\mathbb{R}^{+}} \kappa(A)^{\alpha} \mathrm{e}^{-\alpha \rho(A) s} \mathrm{~d} s \pi(\mathrm{d} A) \\
& =\alpha^{-1} \int_{M_{d}^{-}} \frac{\kappa(A)^{\alpha}}{\rho(A)} \pi(\mathrm{d} A) \\
& <\infty
\end{aligned}
$$

and, thus, $\mathrm{e}^{A s} \in \mathbb{L}^{\alpha}(\lambda \times \pi)$. It is left to show that $\mu_{v}\left(f^{-1}(A, s) \mathbb{R}^{n}\right)=0$ does not hold for $\pi \times \lambda$ almost-every $(A, s)$; however, since

$$
\mu_{v}\left(\mathrm{e}^{-A s} \mathbb{R}^{d}\right)=\mu_{v}\left(\mathbb{R}^{d}\right)
$$

for any $(A, s)$, this follows simply from $\mu_{v}$ being a nonzero measure.

For illustration, let us now calculate the measures $\mu_{X}$ of regular variation in some special cases.

Example 4.1. (Measure of regular variation of OU processes.) SupOU processes with probability measure $\pi$ being a one-point measure (i.e. $\pi(A)=1$ for some $A \in M_{d}^{-}$) are called OU processes and their measure of regular variation is given by

$$
\mu_{X}(B):=\int_{\mathbb{R}^{+}} \mu_{v}\left(\mathrm{e}^{-A s} B\right) \mathrm{d} s .
$$

We consider several examples in the case $d=2$. Let us first assume that the mass of the measure $\mu_{\nu}$ is concentrated on a straight line, i.e. on the points of the form $h=\left(a(1, b)^{\top}\right)_{a \in \mathbb{R} \backslash\{0\}}$ for $b \in \mathbb{R}$; see Figure 4 for an example with $b=0.5$.

1. If $A=c I_{d}, c \in \mathbb{R}^{-}$, is a multiple of the identity matrix then

$$
\mu_{X}(B)=\int_{\mathbb{R}^{+}} \mu_{v}\left(\mathrm{e}^{-c s} B\right) \mathrm{d} s=\int_{\mathbb{R}^{+}} \mathrm{e}^{c \alpha s} \mu_{v}(B) \mathrm{d} s=-\frac{\mu_{v}(B)}{c \alpha} .
$$




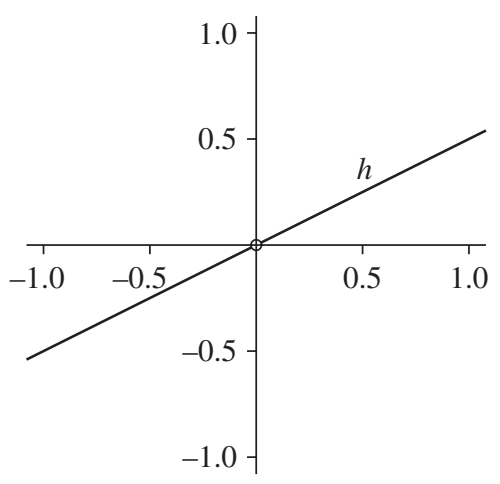

FIGURE 4: Mass of the measures $\mu_{\nu}$ and $\mu_{X}$ in case 1.

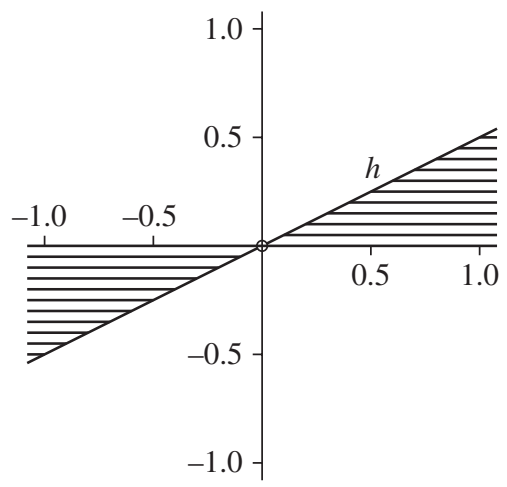

FIGURE 5: Mass of the measure $\mu_{X}$ in case 2.

Consequently, $\mu_{X}$ has its mass in the same direction as $\mu_{v}$ and, thus, its mass is also concentrated on $h$.

2. If $A=\operatorname{diag}\left(a_{1}, a_{2}\right)$ is a diagonal matrix then the mass of $\mu_{X}$ is concentrated on the cones between the straight line $h$ and one of the two axes; see Figure 5. The mass is drawn to the horizontal axis if $a_{2}>a_{1}$, and to the vertical axis if $a_{1}>a_{2}$ (i.e. to the axis associated with the slower exponential decay rate). Intuitively this happens as follows. An extreme jump $\left(x_{1}, x_{2}\right)^{\top}$ occurs at some time $u$ in the past and has direction $s$. This causes an extreme value $\left(\mathrm{e}^{d_{1}(t-u)} x_{1}, \mathrm{e}^{d_{2}(t-u)} x_{2}\right)^{\top}$ at a later time $t$. Since one of the components decays slower, this extreme event is now in a direction closer to the direction with the slowest exponential decay.

3. If $A$ is real diagonalizable, i.e. $A=U D U^{-1}$ with $D=\operatorname{diag}\left(d_{1}, d_{2}\right)$, then the mass is drawn to the eigenspace $e$ that belongs to the biggest eigenvalue $\max \left(d_{1}, d_{2}\right)$. This means that the mass is concentrated on the cone between $e$ and $h$; see Figure 6 . This follows immediately by a change of the basis from the last case.

However, if the support of $\mu_{v}$ is the whole space $\mathbb{R}^{d}$ then the support of $\mu_{X}$ is also $\mathbb{R}^{d}$, regardless of the choice of $A$ in any of the three cases above. 


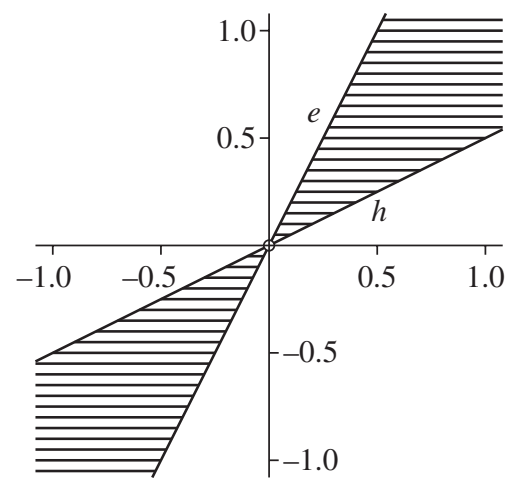

Figure 6: Mass of the measure $\mu_{X}$ in case 3.

As in the general MMA case, we will again have a closer look at the essential condition

$$
\int_{M_{d}^{-}} \frac{\kappa(A)^{\alpha}}{\rho(A)} \pi(\mathrm{d} A)<\infty .
$$

Using the modulus of injectivity, we can derive necessary conditions as in the previous section (see also [3, Proposition 3.3]), where comparable necessary conditions are given for the existence of supOU processes.

Corollary 4.2. Let $\Lambda \in \mathbb{R}^{d}$ be a Lévy basis on $M_{d}^{-} \times \mathbb{R}$ with generating quadruple $(\gamma, \Sigma, \nu, \pi)$, let $v \in \mathrm{RV}\left(\alpha, l, \mu_{\nu}\right)$, and let $X_{0}=\int_{M_{d}^{-}} \int_{\mathbb{R}^{+}} \mathrm{e}^{A s} \Lambda(\mathrm{d} A, \mathrm{~d} s)$ exist following Theorem 4.1. Furthermore, assume that there exist measurable functions $\tau: M_{d}^{-} \mapsto \mathbb{R}^{+} \backslash\{0\}$ and $\vartheta: M_{d}^{-} \mapsto$ $[1, \infty)$ such that

$$
j\left(\mathrm{e}^{A s}\right) \geq \vartheta(A) \mathrm{e}^{-\tau(A) s} \quad \text { for all } s \in \mathbb{R}^{+}, \pi \text {-almost surely. }
$$

Then

$$
\int_{M_{d}^{-}} \frac{\vartheta(A)^{\alpha}}{\tau(A)} \pi(\mathrm{d} A)<\infty
$$

is a necessary condition for $X_{0} \in \mathrm{RV}\left(\alpha, l, \mu_{X}\right)$ with

$$
\mu_{X}(\cdot):=\int_{M_{d}^{-}} \int_{\mathbb{R}^{+}} \int_{\mathbb{R}^{d}} \mathbf{1}_{\{\cdot\}}\left(\mathrm{e}^{A s} x\right) \mu_{v}(\mathrm{~d} x) \mathrm{d} s \pi(\mathrm{d} A) .
$$

Proof. Suppose that

$$
\int_{M_{d}^{-}} \frac{\vartheta(A)^{\alpha}}{\tau(A)} \pi(\mathrm{d} A)=\infty
$$

Then

$$
\begin{aligned}
\int_{M_{d}^{-}} \int_{\mathbb{R}^{+}} j\left(\mathrm{e}^{A s}\right)^{\alpha} \mathrm{d} s \pi(\mathrm{d} A) & \geq \int_{M_{d}^{-}} \int_{\mathbb{R}^{+}} \vartheta(A)^{\alpha} \mathrm{e}^{-\alpha \tau(A) s} \mathrm{~d} s \pi(\mathrm{d} A) \\
& =\alpha^{-1} \int_{M_{d}^{-}} \frac{\vartheta(A)^{\alpha}}{\tau(A)} \pi(\mathrm{d} A) \\
& =\infty .
\end{aligned}
$$

Consequently, $\mathrm{e}^{A s} \notin \mathbb{J}^{\alpha}(\lambda \times \pi)$ and Theorem 3.2 yields the result. 
Finally, as a consequence of Theorem 3.1, we also have regular variation of the process.

Corollary 4.3. Given the conditions of Corollary 4.1, the supOU process $\left(X_{t}\right)_{t \in \mathbb{R}}$ is also regularly varying with index $\alpha$ as a process.

\section{Stochastic volatility model}

\subsection{The model}

In this section we review and analyze the supOU-type stochastic volatility model introduced in [4]. We consider a $d$-dimensional logarithmic stock price process $\left(X_{t}\right)_{t \in \mathbb{R}}$ given by an equation of the form

$$
\mathrm{d} X_{t}=\Sigma_{t}^{1 / 2} \mathrm{~d} W_{t}, \quad X_{0}=0,
$$

where $W$ is a $d$-dimensional Brownian motion and $\Sigma^{1 / 2}$ denotes the unique positive semidefinite square root. The stochastic volatility process $\left(\Sigma_{t}\right)_{t \in \mathbb{R}}$ is given by an $\mathbb{S}_{d}^{+}$-valued supOU process that is independent of the Brownian motion $W$.

Definition 5.1. (Positive semidefinite supOU process.) Let $\Lambda$ be a Lévy basis on $M_{d}^{-} \times \mathbb{R}$ with values in $\mathbb{S}_{d}$. If the process

$$
\Sigma_{t}:=\int_{M_{d}^{-}} \int_{-\infty}^{t} \mathrm{e}^{A(t-s)} \Lambda(\mathrm{d} A, \mathrm{~d} s) \mathrm{e}^{A^{\top}(t-s)}
$$

exists for all $t \in \mathbb{R}$, it is called a positive semidefinite (or $\mathbb{S}_{d}^{+}$-valued) supOU process.

The process $\left(X_{t}\right)_{t \in \mathbb{R}^{+}}$being given by (5.1) with volatility process $\left(\Sigma_{t}\right)_{t \in \mathbb{R}}$ given by a positive semidefinite supOU process is called a multivariate supOU-type stochastic volatility model or SVsupOU.

The introduced model is of course only the most basic version of a SVsupOU. We can easily enhance the model by adding a stochastic or deterministic drift $a$ and a leverage term $\Psi$; see [4] for details. The model is then given by the equation

$$
\mathrm{d} X_{t}=a_{t} \mathrm{~d} t+\Sigma_{t-}^{1 / 2} \mathrm{~d} W_{t}+\Psi\left(\mathrm{d} L_{t}\right), \quad X_{0}=0
$$

where $a$ is an $\mathbb{R}^{d}$-valued predictable process, $W$ is the $d$-dimensional Brownian motion, $L$ is the Lévy process associated with $\Lambda$, and $\Psi: \mathbb{S}_{d} \mapsto \mathbb{R}^{d}$ is a linear operator. The stochastic volatility process $\left(\Sigma_{t}\right)_{t \in \mathbb{R}}$ is again a matrix-valued supOU process. However, the drift term and the leverage term usually dominate the tail behavior, if they are nonvanishing. The leverage term is determined by the behavior of the Lévy process $L$ and, as we always assume the driving Lévy measure to be regularly varying with index $\alpha$, the leverage term is also regularly varying with index $\alpha$. A popular choice for the drift term is

$$
a_{t}=\mu+\beta \Sigma_{t}
$$

where $\beta: \mathbb{S}_{d} \mapsto \mathbb{R}^{d}$ is a linear operator and in this case $a_{t}$ is regularly varying with index $\alpha$, as we will show below. This means that if such a drift or leverage term exists, it dominates the Brownian term, which turns out to be regularly varying with index $2 \alpha$. For this reason, we will only consider the simple model in this section.

Let us start by analyzing the volatility process. Existence of the positive semidefinite supOU processes is given similarly to the existence of $\mathbb{R}^{d}$-valued supOU processes. 
Theorem 5.1. ([3, Theorem 4.1].) Let $\Lambda$ be an $\mathbb{S}_{d}$-valued Lévy basis with generating quadruple $(\gamma, 0, v, \pi)$ and with $\gamma_{0}:=\gamma-\int_{\|x\| \leq 1} x v(\mathrm{~d} x) \in \mathbb{S}_{d}^{+}, v\left(\mathbb{S}_{d} \backslash \mathbb{S}_{d}^{+}\right)=0$,

$$
\int_{\|x\|>1} \ln (\|x\|) v(\mathrm{~d} x)<\infty, \text { and } \int_{\|x\| \leq 1}\|x\| v(\mathrm{~d} x)<\infty .
$$

Furthermore, assume the existence of measurable functions $\rho: M_{d}^{-} \mapsto \mathbb{R}^{+}$and $\kappa: M_{d}^{-} \mapsto$ $[1, \infty)$ such that

$$
\left\|\mathrm{e}^{A s}\right\| \leq \kappa(A) \mathrm{e}^{-\rho(A) s} \quad \text { for all } s \in \mathbb{R}^{+}, \pi \text {-almost surely and } \int_{M_{d}^{-}} \frac{\kappa(A)^{2}}{\rho(A)} \pi(\mathrm{d} A)<\infty .
$$

Then the positive semidefinite supOU process

$$
\Sigma_{t}=\int_{M_{d}^{-}} \int_{-\infty}^{t} \mathrm{e}^{A(t-s)} \Lambda(\mathrm{d} A, \mathrm{~d} s) \mathrm{e}^{A^{\top}(t-s)}
$$

is well defined for all $t \in \mathbb{R}$, has values in $\mathbb{S}_{d}^{+}$for all $t \in \mathbb{R}$, and its distribution is stationary and infinitely divisible. Moreover, the vector representation has the form

$$
\operatorname{vec}\left(\Sigma_{t}\right)=\int_{M_{d}^{-}} \int_{-\infty}^{t} \mathrm{e}^{\left(A \otimes I_{d}+I_{d} \otimes A\right)(t-s)} \operatorname{vec}(\Lambda)(\mathrm{d} A, \mathrm{~d} s) .
$$

Note that in the above theorem $\operatorname{vec}(\Lambda)$ is defined by $\operatorname{vec}(\Lambda)(A):=\operatorname{vec}((\Lambda(A))$ and it is a Lévy basis in $\mathbb{R}^{d^{2}}$.

Based on this theorem, we can now analyze the tail behavior of the volatility process. Therefore, we have to define regular variation in a matrix-valued setting, which is just a translation of $\mathbb{R}^{d}$-valued regular variation. A random matrix $X \in M_{d}$ is said to be regularly varying with index $\alpha>0$ if there exists a slowly varying function $l: \mathbb{R} \mapsto \mathbb{R}$ and a nonzero Radon measure $\mu$ defined on $\mathcal{B}\left(\bar{M}_{d} \backslash\{0\}\right)$ with $\mu\left(\bar{M}_{d} \backslash M_{d}\right)=0$ such that, as $u \rightarrow \infty$,

$$
u^{\alpha} l(u) \mathrm{P}\left(u^{-1} X \in \cdot\right) \stackrel{\mathrm{v}}{\rightarrow} \mu(\cdot)
$$

on $\mathscr{B}\left(\bar{M}_{d} \backslash\{0\}\right)$, and we write $X \in \mathrm{RV}(\alpha, l, \mu)$. Of course, for a random matrix $X \in M_{d}$, there exists the straightforward connection that $X \in \operatorname{RV}(\alpha, l, \mu)$ if and only if $\operatorname{vec}(X) \in \operatorname{RV}\left(\alpha, l, \mu^{v}\right)$, where $\mu^{v}(\operatorname{vec}(A))=\mu(A)$. Given this relationship, we can then analyze the tail behavior of the volatility process, where regular variation can be derived using the results of the previous sections.

Corollary 5.1. Let $\Lambda \in \mathbb{S}_{d}$ be a Lévy basis on $M_{d}^{-} \times \mathbb{R}$ with generating quadruple $(\gamma, 0, v, \pi)$, and let $v \in \operatorname{RV}\left(\alpha, l, \mu_{\nu}\right)$. If the conditions of Theorem 5.1 hold and, additionally,

$$
\int_{M_{d}^{-}} \frac{\kappa(A)^{2 \alpha}}{\rho(A)} \pi(\mathrm{d} A)<\infty
$$

then $\Sigma_{0}=\int_{M_{d}^{-}} \int_{\mathbb{R}^{+}} \mathrm{e}^{A s} \Lambda(\mathrm{d} A, \mathrm{~d} s) \mathrm{e}^{A^{\top} s} \in \mathrm{RV}\left(\alpha, l, \mu_{\Sigma}\right)$ with Radon measure

$$
\mu_{\Sigma}(\cdot):=\int_{M_{d}^{-}} \int_{\mathbb{R}^{+}} \int_{\mathbb{R}^{d}} \mathbf{1}_{\{\cdot\}}\left(\mathrm{e}^{A s} x \mathrm{e}^{A^{\top} s}\right) \mu_{\nu}(\mathrm{d} x) \mathrm{d} s \pi(\mathrm{d} A) .
$$

Furthermore, the supOU process $\left(\Sigma_{t}\right)_{t \in \mathbb{R}}$ is also regularly varying with index $\alpha$ as a process. 
Proof. From Theorem 5.1 we have

$$
\operatorname{vec}\left(\Sigma_{t}\right)=\int_{M_{d}^{-}} \int_{-\infty}^{t} \mathrm{e}^{\left(A \otimes I_{d}+I_{d} \otimes A\right)(t-s)} \operatorname{vec}(\Lambda)(\mathrm{d} A, \mathrm{~d} s),
$$

and, thus, the vectorized volatility process $\operatorname{vec}\left(\Sigma_{t}\right)$ is an MMA process with kernel function $f(A, s)=\mathrm{e}^{\left(A \otimes I_{d}+I_{d} \otimes A\right) s} \mathbf{1}_{[0, \infty)}(s)$. In order to apply Theorem 3.1, it remains to show that $f \in \mathbb{L}^{\alpha}(\lambda \times \pi)$. For that purpose, we make use of the relations $\mathrm{e}^{\left(A \otimes I_{d}+I_{d} \otimes A\right) s}=\mathrm{e}^{A s} \otimes \mathrm{e}^{A s}$ and $\left\|\mathrm{e}^{A s} \otimes \mathrm{e}^{A s}\right\|=\left\|\mathrm{e}^{A s}\right\|^{2}$ (see [14, Chapter 4.2, Problem 28, and Chapter 6.2, Problem 14]) and obtain

$$
\begin{aligned}
\int_{M_{d}^{-}} \int_{\mathbb{R}^{+}}\left\|\mathrm{e}^{\left(A \otimes I_{d}+I_{d} \otimes A\right) s}\right\|^{\alpha} \mathrm{d} s \pi(\mathrm{d} A) & \leq \int_{M_{d}^{-}} \int_{\mathbb{R}^{+}}\left\|\mathrm{e}^{A s}\right\|^{2 \alpha} \mathrm{d} s \pi(\mathrm{d} A) \\
& \leq \int_{M_{d}^{-}} \int_{\mathbb{R}^{+}} \kappa(A)^{2 \alpha} \mathrm{e}^{-2 \alpha \rho(A) s} \mathrm{~d} s \pi(\mathrm{d} A) \\
& =\frac{1}{2 \alpha} \int_{M_{d}^{-}} \frac{\kappa(A)^{2 \alpha}}{\rho(A)} \pi(\mathrm{d} A) \\
& <\infty .
\end{aligned}
$$

Now we want to go one step further and analyze the tail behavior of the logarithmic stock price process. Therefore, we use the independence between $W$ and $\Lambda$, which yields the equality

$$
\int_{0}^{t} \Sigma_{s}^{1 / 2} \mathrm{~d} W_{s} \stackrel{\mathrm{D}}{=}\left(\int_{0}^{t} \Sigma_{s} \mathrm{~d} s\right)^{1 / 2} W_{t} .
$$

We immediately see that it is necessary to analyze the integrated volatility

$$
\Sigma_{t}^{+}:=\int_{0}^{t} \Sigma_{s} \mathrm{~d} s
$$

in order to obtain regular variation of the stock price process. We start with its existence.

Theorem 5.2. ([3, Theorem 4.3].) Let $\Sigma$ be a positive semidefinite supOU process as given in Definition 5.1 that exists according to Theorem 5.1. Then $\Sigma_{t}(\omega)$ is measurable as a function of $t \in \mathbb{R}$ and $\omega \in \Omega$. Also, if

$$
\int_{M_{d}^{-}} \kappa(A)^{2} \pi(\mathrm{d} A)<\infty
$$

then the paths of $\Sigma$ are uniformly bounded in $t$ and the integrated process $\Sigma_{t}^{+}$exists for all $t \in \mathbb{R}^{+}$.

Another important and closely related characteristic of a time series is the discrete-time process of log returns observed over time periods of length $\Delta \in \mathbb{R}^{+}$(representing, for example, observation intervals, trading periods, etc.) and given by

$$
Z_{n}:=X_{n \Delta}-X_{(n-1) \Delta}=\int_{(n-1) \Delta}^{n \Delta} \Sigma_{s}^{1 / 2} \mathrm{~d} W_{s} \stackrel{\mathrm{D}}{=}\left(\int_{(n-1) \Delta}^{n \Delta} \Sigma_{s} \mathrm{~d} s\right)^{1 / 2} W_{\Delta} .
$$

Existence of the related integrated volatilities

$$
\Sigma_{n}^{+}:=\int_{(n-1) \Delta}^{n \Delta} \Sigma_{s} \mathrm{~d} s
$$


is given by Theorem 5.1, and conditions for regular variation of $\Sigma_{n}^{+}$and of the integrated volatility $\Sigma_{t}^{+}$can be derived simultaneously.

Corollary 5.2. Let $\Lambda \in \mathbb{S}_{d}$ be a Lévy basis on $M_{d}^{-} \times \mathbb{R}$ with generating quadruple $(\gamma, 0, v, \pi)$, and let $v \in \operatorname{RV}\left(\alpha, l, \mu_{v}\right)$. If the conditions of Theorem 5.2 hold and, additionally,

$$
\int_{M_{d}^{-}} \frac{\kappa(A)^{2 \alpha}}{\rho(A)^{\alpha+1}} \pi(\mathrm{d} A)<\infty
$$

then $\Sigma_{n}^{+} \in \operatorname{RV}\left(\alpha, l, \mu_{\Sigma_{n}^{+}}\right)$with Radon measure

$$
\begin{aligned}
\mu_{\Sigma_{n}^{+}}(B):=\int_{M_{d}^{-}} \int_{\mathbb{R}} \int_{\mathbb{R}^{d}} \mathbf{1}_{B}\left(\int_{u \vee(n-1) \Delta}^{n \Delta} \mathrm{e}^{A(s-u)} x \mathrm{e}^{A^{\top}(s-u)} \mathbf{1}_{(-\infty, n \Delta]}(u) \mathrm{d} s\right) \\
\times \mu_{v}(\mathrm{~d} x) \mathrm{d} u \pi(\mathrm{d} A)
\end{aligned}
$$

and $\Sigma_{t}^{+} \in \mathrm{RV}\left(\alpha, l, \mu_{\Sigma_{t}^{+}}\right)$with

$$
\mu_{\Sigma_{t}^{+}}(B):=\int_{M_{d}^{-}} \int_{\mathbb{R}} \int_{\mathbb{R}^{d}} \mathbf{1}_{B}\left(\int_{u \vee 0}^{t} \mathrm{e}^{A(s-u)} x \mathrm{e}^{A^{\top}(s-u)} \mathbf{1}_{(-\infty, t]}(u) \mathrm{d} s\right) \mu_{\nu}(\mathrm{d} x) \mathrm{d} u \pi(\mathrm{d} A) .
$$

Furthermore, the process $\left(\Sigma_{t}^{+}\right)_{t \in \mathbb{R}^{+}}$is also regularly varying with index $\alpha$ as a process.

Proof. Again, we use the vector representation of the process and, from the proof of Theorem 3.12 of [3], obtain

$$
\begin{aligned}
\operatorname{vec}\left(\Sigma_{n}^{+}\right) & =\int_{(n-1) \Delta}^{n \Delta} \int_{M_{d}^{-}} \int_{-\infty}^{s} \mathrm{e}^{\left(A \otimes I_{d}+I_{d} \otimes A\right)(s-u)} \operatorname{vec}(\Lambda)(\mathrm{d} A, \mathrm{~d} u) \mathrm{d} s \\
& =\int_{\mathbb{R}} \int_{M_{d}^{-}} g(A, t, u) \operatorname{vec}(\Lambda)(\mathrm{d} A, \mathrm{~d} u)
\end{aligned}
$$

with

$$
g(A, t, u):=\int_{u \vee(n-1) \Delta}^{n \Delta} \mathrm{e}^{\left(A \otimes I_{d}+I_{d} \otimes A\right)(s-u)} \mathbf{1}_{(-\infty, n \Delta]}(u) \mathrm{d} s .
$$

As before, it remains to show that $g(A, t, u) \in \mathbb{L}^{\alpha}(\lambda \times \pi)$ in order to apply Theorem 3.1. Therefore, we estimate

$$
\begin{aligned}
\|g(A, t, u)\| & \leq \mathbf{1}_{(-\infty, n \Delta]}(u) \int_{u \vee(n-1) \Delta}^{n \Delta}\left\|\mathrm{e}^{\left(A \otimes I_{d}+I_{d} \otimes A\right)(s-u)}\right\| \mathrm{d} s \\
& \leq \mathbf{1}_{(-\infty, n \Delta]}(u) \int_{u \vee(n-1) \Delta}^{n \Delta} \kappa(A)^{2} \mathrm{e}^{-2 \rho(A)(s-u)} \mathrm{d} s \\
& =\frac{\kappa(A)^{2}}{-2 \rho(A)}\left(\mathrm{e}^{-2 \rho(A)(n \Delta-u)} \mathbf{1}_{(-\infty, n \Delta]}(u)-\mathbf{1}_{((n-1) \Delta, n \Delta)}(u)\right. \\
& \left.-\mathrm{e}^{-2 \rho(A)((n-1) \Delta-u)} \mathbf{1}_{(-\infty,(n-1) \Delta]}(u)\right)
\end{aligned}
$$

For the first term of the sum, we obtain

$$
\int_{M_{d}^{-}} \int_{-\infty}^{n \Delta}\left|\frac{\kappa(A)^{2} \mathrm{e}^{-2 \rho(A)(n \Delta-u)}}{-2 \rho(A)}\right|^{\alpha} \mathrm{d} u \pi(\mathrm{d} A)=\frac{1}{2^{\alpha+1} \alpha} \int_{M_{d}^{-}} \frac{\kappa(A)^{2 \alpha}}{\rho(A)^{\alpha+1}} \pi(\mathrm{d} A)<\infty .
$$


The second summand is in $\mathbb{L}^{\alpha}(\lambda \times \pi)$, since the function has bounded support, and, for the last term in the sum, we simply substitute $n$ by $n-1$ in the first term. The result for $\Sigma_{t}^{+}$follows directly setting $\Delta=t$ and $n=1$.

Note that this result also gives us regular variation with index $\alpha$ of a possible drift term $a_{t}=\mu+\beta \Sigma_{t}$.

The next step is to derive the tail behavior of the square root $\left(\Sigma^{+}\right)^{1 / 2}$ of the integrated volatility process.

Lemma 5.1. Let $\Sigma$ be a random variable with values in $\mathbb{S}_{d}^{+}$, and let $\Sigma^{1 / 2}$ be its square root. Then $\Sigma \in \operatorname{RV}\left(\alpha, l, \mu_{\Sigma}\right)$ if and only if $\Sigma^{1 / 2} \in \operatorname{RV}\left(2 \alpha, l^{1 / 2}, \mu_{\Sigma}^{1 / 2}\right)$ with $l^{1 / 2}(x):=l\left(x^{2}\right)$ and $\mu_{\Sigma}^{1 / 2}(B):=\mu\left(B^{2}\right)$.

Proof. Note that the square root of a matrix in $\mathbb{S}_{d}^{+}$is a bijective mapping and is thus well defined. Since both functions, the square as well as the square root, map compacts to compacts, we can apply Proposition 3.18 of [26].

Now we can consider the log-returns and the logarithmic stock price process regarding their tail behavior.

Theorem 5.3. Let $\left(X_{t}\right)_{t \in \mathbb{R}}$ be the stock price process given by (5.1), let $Z_{n}$ be the log-returns given by (5.3), and let $\Sigma_{n}^{+}$be the increments of a positive semidefinite supOU process $\left(\Sigma_{t}\right)_{t \in \mathbb{R}^{+}}$. Furthermore, let the conditions of Corollary 5.2 hold. Then $Z_{n} \in \operatorname{RV}\left(2 \alpha, l^{1 / 2}, \mu_{Z}\right)$ with Radon measure

$$
\mu_{Z}(B):=\mathrm{E}\left(\mu_{\Sigma_{n}^{+}}^{1 / 2}\left(W_{\Delta}^{-1}(B)\right)\right)
$$

and $X_{t} \in \mathrm{RV}\left(2 \alpha, l^{1 / 2}, \mu_{X}\right)$ with

$$
\mu_{X}(B):=\mathrm{E}\left(\mu_{\Sigma_{t}^{+}}^{1 / 2}\left(W_{t}^{-1}(B)\right)\right)
$$

where $W_{\Delta}: M_{d}^{-} \mapsto \mathbb{R}^{d}$ is considered to be a random linear mapping with $W_{\Delta}(x):=x W_{\Delta} \stackrel{\mathrm{D}}{=}$ $\Delta x N\left(0, I_{d}\right)$ (likewise for $\left.W_{t}\right)$. Furthermore, $\left(X_{t}\right)_{t \in \mathbb{R}}$ is also regularly varying with index $2 \alpha$ as a process.

Proof. Since $W$ and $\Lambda$ are independent, we have

$$
Z_{n} \stackrel{\mathrm{D}}{=}\left(\int_{(n-1) \Delta}^{n \Delta} \Sigma_{s} \mathrm{~d} s\right)^{1 / 2} W_{\Delta} \quad \text { and } \quad \int_{0}^{t} \Sigma_{s}^{1 / 2} \mathrm{~d} W_{s} \stackrel{\mathrm{D}}{=}\left(\int_{0}^{t} \Sigma_{s} \mathrm{~d} s\right)^{1 / 2} W_{t} .
$$

From Corollary 5.2 we know that

$$
\Sigma_{n}^{+} \in \mathrm{RV}\left(\alpha, l, \mu_{\Sigma_{n}^{+}}\right) \quad \text { and } \quad \Sigma_{t}^{+} \in \mathrm{RV}\left(\alpha, l, \mu_{\Sigma_{t}^{+}}\right),
$$

and this, together with Lemma 5.1, yields

$$
\left(\Sigma_{n}^{+}\right)^{1 / 2} \in \mathrm{RV}\left(2 \alpha, l^{1 / 2}, \mu_{\Sigma_{n}^{+}}^{1 / 2}\right) \quad \text { and } \quad\left(\Sigma_{t}^{+}\right)^{1 / 2} \in \mathrm{RV}\left(2 \alpha, l^{1 / 2}, \mu_{\Sigma_{t}^{+}}^{1 / 2}\right)
$$

Finally, we use the fact that the Brownian motion has finite moments to apply the multivariate version of Breiman's lemma (see [5, Proposition A.1]), which yields the result. 


\subsection{Relevance and applications in finance}

Let us conclude with some final remarks. First, we easily see that the model allows for heavy tails, in the volatility as well as in logarithmic stock prices and log-returns. This is a useful fact, since observed marked data often shows heavy tails. Furthermore, we see that there is a direct connection between the indexes of regular variation of the driving Lévy measure on the one hand and the volatility, log-prices, and log-returns on the other hand. We can also calculate the concrete measure $\mu$ of regular variation in order to describe the spatial structure of the extremes.

Second, we note that all the results given above hold also in the case of an OU-type stochastic volatility model, where the volatility is modeled by an $\mathbb{S}_{d}^{+}$-valued OU process. This is obvious, since OU processes are special cases of supOU processes with $\pi$ being a Dirac measure.

In a financial context, the results can now be used for a statistical analysis of observed data. We can use one of the well-established estimators (see [9, Section 6] or [27, Section 4]) to estimate the index of regular variation of the given data (logarithmic stock prices or log-returns). The result can then be compared with the estimated index of regular variation of the integrated volatility. If they do not match by the factor of 2 , this is a hint for the existence of a leverage or drift term of the form specified in this paper. Yet, there is still some future work to be done to analyze and estimate the (index of regular variation of the) integrated volatility, since it cannot be observed directly. If we make additional assumptions on the different terms (leverage, drift) to exist or not, we can calculate the index of regular variation of the log-prices or log-returns from the index of the volatility and vice versa.

It would also be very interesting to generalize the stochastic volatility model by substituting the Brownian motion $W_{t}$ by a more general Lévy process $\widetilde{L}_{t}$. However, as there is then no analogue of (5.2) available, it will be much more difficult to get results for this case and different methods will be needed.

\subsection{Modelling the correlation breakdown}

Applied research in financial mathematics and econometrics has often noted that one typically encounters what has been dubbed 'correlation breakdown' in times of severe crisis. This notion means that when extreme negative events potentially affecting the whole (or large parts of the) economy occur, basically all traded stocks are loosing tremendously in value simultaneously and the correlations between them are seemingly more or less 1 . Moreover, after such an event the variances are typically extremely high. Models employed in financial institutions (for risk management) clearly need to include this feature in order to be realistic and provide accurate predictions.

Our results on the (sup)OU stochastic volatility model allow us to understand how to incorporate such effects into the model. Clearly, the most extreme movements in crisis can typically not come from the Brownian term but have to come from jumps. Hence, $\Psi$ needs to be chosen nonzero and such that when all variances have a jump upwards, all prices have jumps downward. Assume now that the positive semidefinite Lévy basis is regularly varying with index $\alpha$, and the measure $\mu_{v}$ is concentrated on the rank-one matrices with all diagonal elements being nonzero and all correlations being 1 . The extremes of $\Sigma$ are now caused by a single big jump which will be such that it is (almost) a rank-one matrix with all diagonal elements being nonzero. After the occurrence of the jump the process $\Sigma$ will be almost equal to the value of this jump and, hence, all correlations will be very close to 1 for quite some time afterwards. Moreover, by the choice of parameters, the prices will simultaneously have a huge jump downwards. Clearly, this would model the 'correlation breakdown'. Our results actually 
show that $\Sigma$ would be regularly varying with index $\alpha$ and $\mu_{\Sigma}$ would be concentrated on the rank-one matrices with all diagonal elements being nonzero as this class of matrices is preserved by the mappings $X \mapsto \mathrm{e}^{A s} X \mathrm{e}^{A^{\top} s}$ for all $A \in M_{d}(\mathbb{R})$ and $s \in \mathbb{R}^{+}$. Likewise, the log-prices would be regularly varying with index $\alpha$ (unless we had a drift with heavier tails which seems unreasonable) and the measure of regular variation for the log-prices follows easily, because what matters is only the linear transformation $\Psi$ of the driving positive semidefinite Lévy basis. Note that the measure of regular variation of the log-prices will in general still have a completely nondegenerate support (in the positive $d$-dimensional cone).

In practice, the above explained model can only form an important building block of a realistic and suitable model, since not all extreme events affect the whole economy, some only affect individual sectors of industry or companies. However, for such extensions (which basically demand regular variation of $\Lambda$ with appropriate $\mu_{\Lambda}$ ), our results provide the necessary insight into the resulting tail behavior.

\section{Acknowledgements}

We thank the Editor and an anonymous referee for helpful remarks that improved the paper. Furthermore, both authors gratefully acknowledge the support of the Technische Universität München - Institute for Advanced Study, funded by the German Excellence Initiative. Additionally, the first author acknowledges the support of the International Graduate School of Science and Engineering (IGSSE) at Technische Universität München.

\section{References}

[1] Barndorff-Nielsen, O. E. (2001). Superposition of Ornstein-Uhlenbeck type processes. Theory Prob. Appl. 45, 175-194.

[2] Barndorff-Nielsen, O. E. and Shephard, N. (2001). Non-Gaussian Ornstein-Uhlenbeck-based models and some of their uses in financial economics (with discussion). J. R. Statist. Soc. B 63, 167-241.

[3] Barndorff-Nielsen, O. E. And Stelzer, R. (2011). Multivariate supOU processes. Ann. Appl. Prob. 21, 140-182.

[4] Barndorff-Nielsen, O. E. And Stelzer, R. (2011). The multivariate supOU stochastic volatility model. To appear in Math. Finance.

[5] BasraK, B., Davis, R. A. And Mikosch, T. (2002). Regular variation of GARCH processes. Stoch. Process. Appl. 99, 95-115.

[6] Bender, C., Lindner, A. And Schicks, M. (2011). Finite variation of fractional Lévy processes. To appear in J. Theoret. Prob.

[7] Brockwell, P. J. (2001). Lévy-driven CARMA processes. Ann. Inst. Statist. Math. 53, 113-124.

[8] Brockwell, P. J. (2004). Representations of continuous-time ARMA processes. In Stochastic Methods and Their Applications (J. Appl. Prob. Spec. Vol. 41A), eds J. Gani and E. Seneta, Applied Probability Trust, Sheffield, pp. 375-382.

[9] Embrechts, P., KlüPpelberg, C. And Mikosch, T. (1997). Modelling Extremal Events. For Insurance and Finance. Springer, Berlin.

[10] FASEn, V. (2005). Extremes of regularly varying Lévy-driven mixed moving average processes. Adv. Appl. Prob. 37, 993-1014.

[11] FASEN, V. (2009). Extremes of Lévy driven mixed MA processes with convolution equivalent distributions. Extremes 12, 265-296.

[12] FASEN, V. AND KLÜPPElberg, C. (2007). Extremes of supOU processes. In Stochastic Analysis and Applications (Abel Symp. 2), eds F. Benth et al., Springer, Berlin, pp. 339-359.

[13] Griffin, J. E. And SteEl, M. F. J. (2010). Bayesian inference with stochastic volatility models using continuous superpositions of non-Gaussian Ornstein-Uhlenbeck processes. Comput. Statist. Data Anal. 54, 2594-2608.

[14] Horn, R. A. And Johnson, C. R. (1991). Topics in Matrix Analysis. Cambridge University Press.

[15] Hult, H. And LindsKog, F. (2005). Extremal behavior of regularly varying stochastic processes. Stoch. Process. Appl. 115, 249-274.

[16] Hult, H. And Lindskog, F. (2006). On regular variation for infinitely divisible random vectors and additive processes. Adv. Appl. Prob. 38, 134-148. 
[17] Jacobsen, M., Mikosch, T., Rosiński, J. And SAMorodnitsky, G. (2009). Inverse problems for regular variation of linear filters, a cancellation property for $\sigma$-finite measures and identification of stable laws. Ann. Appl. Prob. 19, 210-242.

[18] LindsKog, F. (2004). Multivariate extremes and regular variation for stochastic processes. Doctoral Thesis, ETH Zurich.

[19] MarquardT, T. (2006). Fractional Lévy processes with an application to long memory moving average processes. Bernoulli 12, 1099-1126.

[20] Marquardt, T. (2007). Multivariate fractionally integrated CARMA processes. J. Multivariate Anal. 98, 17051725.

[21] Marquardt, T. and Stelzer, R. (2007). Multivariate CARMA processes. Stoch. Process. Appl. 117, $96-120$.

[22] Pedersen, J. (2003). The Lévy-Ito decomposition of an independently scattered random measure. MaPhySto Res. Rep. 2, MaPhySto, University of Aarhus. Available at http://www.maphysto.dk/.

[23] Pigorsch, C. and Stelzer, R. (2009). A multivariate Ornstein-Uhlenbeck type stochastic volatility model. Submitted.

[24] Rajput, B. S. AND Rosiński, J. (1989). Spectral representations of infinitely divisible processes. Prob. Theory Relat. Fields 82, 451-487.

[25] ReSnick, S. I. (1986). Point processes, regular variation and weak convergence. Adv. Appl. Prob. 18, 66-138.

[26] Resnick, S. I. (1987). Extreme Values, Regular Variation, and Point Processes. Springer, New York.

[27] Resnick, S. I. (2007). Heavy-Tail Phenomena. Probabilistic and Statistical Modeling. Springer, New York.

[28] Samorodnitsky, G. and TaquU, M. S. (1994). Stable Non-Gaussian Random Processes. Chapman \& Hall, New York.

[29] Sato, K.-I. (2002). Lévy Processes and Infinitely Divisible Distributions. Cambridge University Press.

[30] Surgailis, D., Rosiński, J., Mandrekar, V. and Cambanis, S. (1993). Stable mixed moving averages. Prob. Theory Relat. Fields 97, 543-558. 